\title{
Influence of Core Swirling Motion on Aerodynamic Performances of Lobed Exhaust Nozzle
} Yulin Ding* and Youhong Liu† and Liwei Du**

\author{
*Ph.D. Candidate, School of Energy and Power Engineering, Beihang University, Beijing, \\ 100191, People's Republic of China (Corresponding Author) \\ $\dagger$ Professor, School of Energy and Power Engineering, Beihang University, Beijing, 100191, \\ People's Republic of China \\ ** Ph.D., Aero Engine Corporation of China, Beijing, 101304, People's Republic of China \\ *Corresponding: yulinding@126.com
}

\begin{abstract}
Influence of core flow inlet swirl angle on aerodynamic performances of an exhaust nozzle with scarfed lobed mixer was studied by the validated computational approach. The computational simulation was conducted by resolving the steady form of discretized threedimensional Reynolds Averaged Navier-Stokes equations with the shear stress transport $\mathrm{k}-\Omega$ turbulence model. Simulation results depict that swirling motions have ignorable influence on the flow field of the top part in the cross sections slightly downstream of the lobed trailing edge. Besides, for the flow field downstream of the $\mathrm{L} / \mathrm{D}=0.1$ cross section, the swirling motions are suggested to cause the clockwise stream-wise vortex to stretch into several smaller-scale vortexes. When the case with a bigger swirling angle is investigated, the induced smaller-scale vortexes are more strengthened by the swirling motions. Concerning the $15^{\circ}$ swirling case, the loss caused by the destroyed vortex pattern and the benefit induced by the improved smaller-scale vortexes almost counteract with each other with respect to the thermal mixing efficiency. In the last studied cross section as compared with the baseline case, the case with a maximum swirling angle of $30^{\circ}$ has increased $6.94 \%$ for the thermal mixing efficiency and decreased $0.42 \%$ for the total pressure recovery coefficient.
\end{abstract}

\section{Keywords}

swirling flow; lobed nozzle; stream-wise vortex; mixing efficiency; total pressure loss

\section{Nomenclature}

$\begin{array}{ll}C_{C C W} & \text { counter-clockwise circulation } \\ C_{C W} & \text { clockwise circulation } \\ C_{p} & \text { specific heat at constant pressure, } \mathrm{J} /(\mathrm{kg} \cdot \mathrm{K}) \\ D & \text { bypass diameter at inlet cross section of exhaust nozzle, } \mathrm{m} \\ \text { NORDIS } & \begin{array}{l}\text { normalized mixing distance downstream of the lobed mixer incorporated in } \\ \text { computational method validation }\end{array} \\ L & \text { distance between lobe trailing edge plane and studied plane along the axial } \\ & \text { direction, } \mathrm{m} \\ \dot{m} & \text { mass flow rate, } \mathrm{kg} / \mathrm{s} \\ p^{*} & \text { total pressure, } \mathrm{Pa} \\ T & \text { static temperature, } \mathrm{K} \\ u, v, w & \text { component of velocity in } \mathrm{X}, \mathrm{Y}, \text { and } \mathrm{Z} \text { coordinate, } \mathrm{m} / \mathrm{s} \\ X, Y, Z & \text { three directions of Cartesian coordinate system, } \mathrm{m} \\ y^{+} & \text {normalized first grid point distance away from the wall boundary }\end{array}$


$\eta$

$\sigma$

$\omega$

\section{Subscript}

c-mix

core

fan

ideal-mix

in

$\operatorname{mix}$

real

STV thermal mixing efficiency

total pressure recovery coefficient

normalized vortex

complete mixing between core flow and bypass flow

core flow

bypass flow

one-dimensional ideal expansion process for the ideally mixed flow

inlet cross section of exhaust nozzle

non-complete mixing between core flow and bypass flow

real working condition for exhaust nozzle

stream-wise vortex

\section{Introduction}

Mixing process within the exhaust nozzle of a turbofan engine is suggested to be of great importance because mixing efficiency, total pressure loss and thrust of the exhaust nozzle are highly influenced by the detailed mixing process. According to previous research results [1], the lobed mixer device has been widely used to improve the mixing process. The lobed mixer is obtained by shaping the conventional splitter mixer into the lobe-like structure. When a conventional splitter mixer is replaced by a lobed mixer, mixing efficiency of the mixed flow is found to be raised apparently [2]. Moreover, both the infrared signal and the aero-acoustic noise are suggested to be alleviated with the usage of a well-designed lobed mixer [3][4]. However, due to the enhanced mixing process downstream of the lobed trailing edge, more energy losses are required for the mixing process. Based on previous investigation conclusions, the benefits achieved by utilizing a lobed mixer are due to three primary reasons [5]: the large-scale stream-wise vortexes downstream of the lobed mixer caused by the lobe-like surface, the increased length of the shear mixing perimeter and the azimuthal vortexes occurring in the boundary between the core and bypass flow attracted by the essential Kelvin-Helmholtz (K-H) instability.

Numerous researchers have completed a variety of studies to reveal the underlying mechanism of the forced mixing process within the lobed exhaust nozzle. Skebe and Paterson [6] performed an experiment to study the forced mixing process with the usage of Laser-Doppler Anemometer (LDA). Their experimental efforts manifested that the complicated flow field within the lobed mixer was predominated by the inviscid flow motions which resulted in the influence of boundary layer confined to the region approximating the lobe surface. Moreover, Skebe and Paterson drew the conclusion that the large-scale stream-wise vortex arrays originating from the lobed mixer were mainly inviscid. Waitz [7] experimentally studied the enhanced mixing process with the existence of large-scale stream-wise vortexes. And their findings suggested that the obvious enhancement of mixing efficiency was directly caused by the strain rate field associated with the large-scale streamwise vortexes that resulting in both the increased interfacial areas and the enhanced local gradients of fluid properties. Through introducing Hot-wire Anemometer (HWA) for the spectrum analyzing and LDA for the measurement of large-scale stream-wise vortexes, Mao and Yu [8] studied both K$\mathrm{H}$ vortexes and large-scale stream-wise vortexes in the wake of a lobed mixer with only one lobe incorporated in the geometrical model. They found that the K-H vortexes in the wake of the studied 
lobed mixer were characterized by two primary frequencies and the frequencies were depicted to be higher than those for the case with conventional splitter mixer. Their measurements also demonstrated that the $\mathrm{K}-\mathrm{H}$ vortexes served as an essential role in producing the turbulent kinetic energy than the large-scale stream-wise vortexes. And the induced large-scale stream-wise vortexes were concluded to be responsible for the accelerated breakdown process of the $\mathrm{K}-\mathrm{H}$ vortexes.

Except for the conducted investigations concerning the mechanism of the forced mixing process, lots of researchers have devoted their efforts in revealing the influence of geometrical lobe parameters on both the characteristics of the mixed flow field and the aerodynamic performance of the lobed nozzle. A lobed mixer is mainly defined by several essential geometrical parameters, i.e. lobe number, lobe length, inward penetrating angle, outward penetrating angle and lobe width. In addition, both scarfing and scalloping optimizations can exert distinct influences on the geometry of the lobed mixer. Tsui and $\mathrm{Wu}$ [9] conducted numerical investigations to study the impact of detailed lobe geometries on the mixing performances through the finite-volume approach with the second-order difference scheme. Their numerical research results showed that the convex lobe contour would generate the vortexes with augmented magnitudes. Besides, it can be concluded that when the lobe trailing edge is shaped into the form of a sinusoidal curve with broad trough region, the lobe-induced large-scale stream-wise vortexes would be well organized and thus leading to a mixed state with desired mixing efficiency. Yu and Yip [10] measured the mixed flow field by using a two-component fiber-optic laser Doppler anemometer and studied the effect of lobe scalloping on the forced mixing characteristics within the lobed nozzle. Their experimental measurements depicted that the magnitude of the large-scale stream-wise vortex adjacent to the lobe trailing edge of the scalloped case was higher than that for the case without scalloping modification. This finding was due to the fact that one additional pair of stream-wise vortexes was formed for the scalloped case. Their experimental results also confirmed that the scalloping modification would make the mixing process more rapid. Except for the investigations above, Liu and Xie have also conducted a series of meaningful studies concerning the effect of detailed lobe geometrical parameters on the aerodynamic performance of the life-sized lobed nozzle, i.e. lobe number [11], lobe width [12] and forced mixing length [13].

As for the studied lobed nozzle, core flow inlet swirl is indicated to affect the development process of the large-scale stream-wise vortexes and thus have an impact on the aerodynamic performance of the lobed nozzle. According to published articles, some research efforts have been devoted to study the effect of the swirl flow on aerodynamic performance of the exhaust nozzle. Mager [14] et al. conducted a numerical study to investigate the swirl flow within a nozzle with the assumption of the incompressible flow. They found that under the condition of weak swirling motions a mass-dominated core structure showed up, and the mass-dominated core structure was mainly unaffected by the swirling motions. Based on the researches concerning swirling flows within an annular exhaust nozzle, Hoffman and Thompson [15] quantitatively examined the effect of four swirl-induced types on both the specific impulses and discharge coefficients. It is noted that the investigated four swirl-induced types were the constant angle, free vortex, forced vortex and Rankine vortex. Dutton [16] revealed that swirling motions had a minor influence on the specific impulse efficiency while the discharge coefficient declined significantly for the swirling cases. Dutton also suggested that as for both the converging-diverging and converging exhaust nozzle, the velocity near the centerline was enhanced as a result of swirling motions. Knowles and Carpenter [17] developed a quasi-cylindrical theory for the subcritical swirling flow within annular nozzles 
and the influence of swirling velocity profiles on the mass flow rate, impulse function and nozzle exhaust thrust was surveyed. Moreover, they reported that the specific thrust of the investigated exhaust nozzle was abated under the condition of swirling flows and the abating rate was obviously affected by velocity profiles of swirling motions.

With regard to the surveys concerning the influence of core flow inlet swirl on aerodynamic performances of the lobed nozzle, Mahallati and Lei accomplished some thoughtful investigations. Mahallati and Lei [18] experimentally investigated the development process of core flow inlet swirl motions and some fundamental conclusions were acquired. As for the testing apparatus used by Mahallati and Lei, the swirl motions were induced by swirl vanes with a uniform swirl angle of $0^{\circ}$, $5^{\circ}, 10^{\circ}, 20^{\circ}$, and $30^{\circ}$, respectively. Under the condition of high swirling angles, most of the core flow inlet swirl motions between the lobe trough and crest were removed by the lobe surface while the swirl motions in the core district were not distinctly influenced by the lobe surface. Their investigations also suggested that the swirl motions induced a region downstream of the plug body, which was characterized by the reversed flow. In 2012, Mahallati and Lei [19] performed another experiment to study the influence of the core flow inlet swirl on the forced mixing process caused by a lobed mixer with scalloping modification. Their measurements verified that as for the cases with a swirl angle less than $10^{\circ}$, the forced mixing process was strengthened at the expense of minimal thrust loss. Additionally, under the condition of the increased swirl angle, downstream mixing was enhanced by the rapid interaction among various vortexes within the mixing flow. Taking the experimental investigations conducted by Mahallati and Lei into consideration, it should be noted that the tested lobed mixer in their experiments was a scaled-down lobed mixer.

This paper presents a detailed numerical investigation concerning the influence of the core flow inlet swirl on aerodynamic performances of a lobed nozzle. The lobed nozzle studied in this article is life-sized, and the incorporated lobed mixer is a scarfed lobed mixer with 18 lobes. Simulation results are achieved by solving the three-dimensional steady Reynolds-Averaged NavierStokes (RANS) equations. During numerical investigations, the core flow inlet swirl is induced by a constant swirl angle of $0^{\circ}, 5^{\circ}, 10^{\circ}, 15^{\circ}, 20^{\circ}, 25^{\circ}$ and $30^{\circ}$ at the inlet cross section of the core flow. Based on predicted results, the influence of the core swirling flow on the mixing flow field is discussed and both the mixing efficiency and total pressure loss of the lobed nozzle for all the studied cases are quantitatively studied.

\section{Numerical simulation}

\subsection{Investigated geometry}

The geometrical model explored in this paper is a life-sized exhaust nozzle with a scarfed lobed mixer installed between the core and bypass flow. The investigated nozzle is a converging axisymmetric nozzle. A plug body is positioned in the center region of the exhaust nozzle to avoid large-scale recirculation regions caused by the sudden expansion of the core flow. Because both the exhaust nozzle and the lobed mixer incorporated in this study are axisymmetric structures, only a small portion covering one lobe body is selected as the computational domain. In order to predict the flow field with high precision, the mixing process between the exhausting flow and the ambient flow is also incorporated in the study. Fig.1 shows the computational domain included in the numerical studies. At the entrance of the bypass flow the shroud diameter is D and all the remaining dimensions used in this paper are all normalized by the magnitude of D. Detailed normalized dimensions of the exhaust nozzle are presented in Fig.2. As for the ambient flow field, the radius 
and the axial length are $2.8 \mathrm{D}$ and $16 \mathrm{D}$, respectively.

The axisymmetric lobed mixer used in this investigation consists of 18 lobes with each lobe body covering a small section of $20^{\circ}$ along the circumference direction. Fig. 3 presents detailed views of the studied lobed mixer and Tab.1 presents the normalized dimensional parameters that outline the configuration of the un-scarfed lobed mixer. It is noted that the tested lobed mixer is a scarfed lobed mixer with a scarfing angle of $10^{\circ}$ as depicted in Fig.3(b).

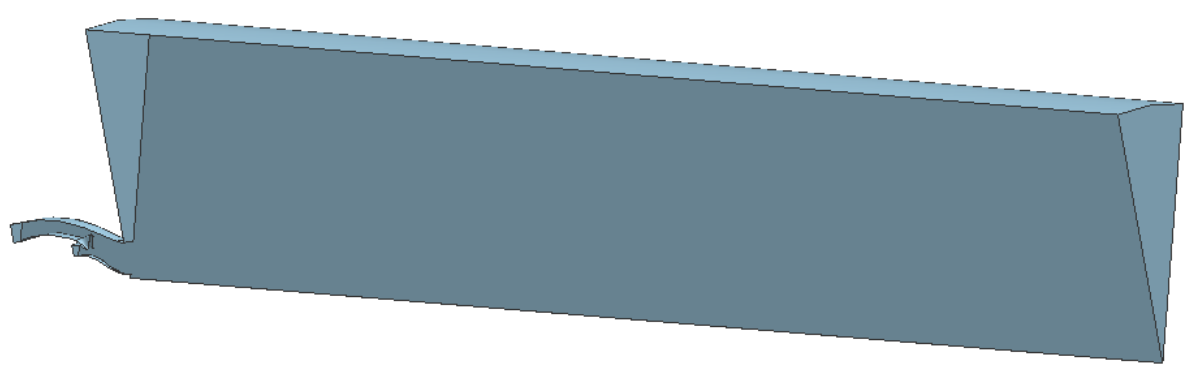

(a) Computational domain of the ambient flow field

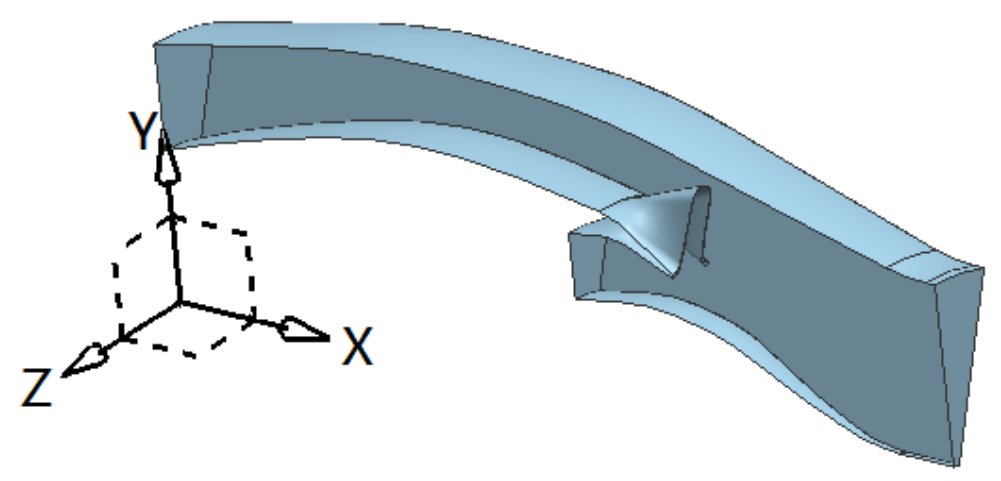

(b) Computational domain of the lobed nozzle

Fig.1 Computational domain of the investigated geometrical model

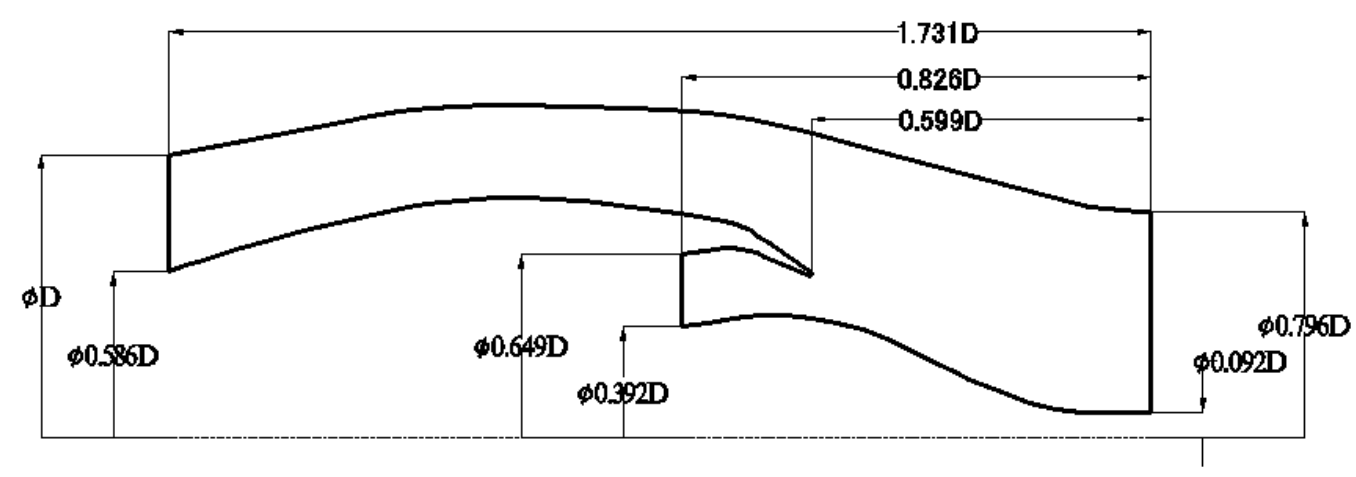

Fig.2 Schematic of the exhaust nozzle 


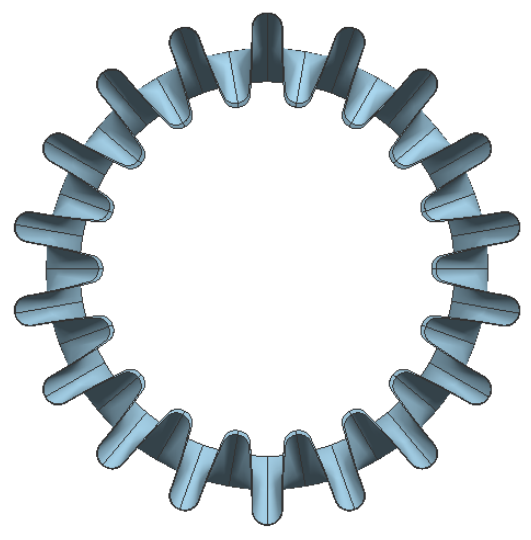

(a) Lobed mixer body

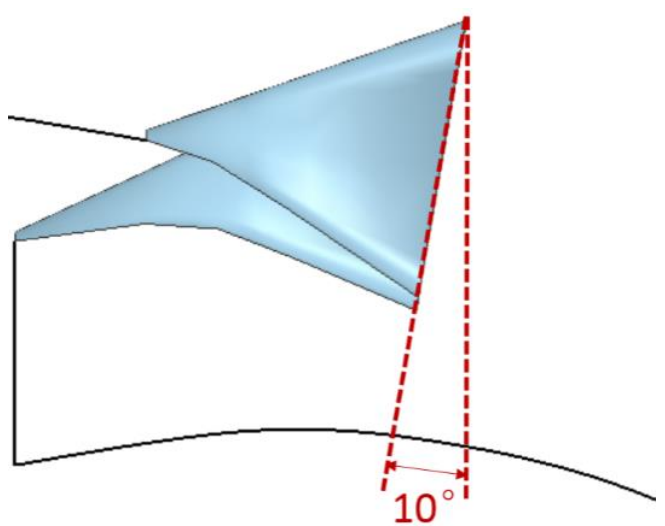

(b) Scarfing angle

Fig.3 Configuration of the scarfed lobed mixer

\begin{tabular}{|c|c|c|}
\hline \multicolumn{2}{|c|}{ Tab.1 Detailed dimensions of the lobed mixer } \\
\hline Lobe parameters & $\begin{array}{c}\text { Values } \\
\text { (measured by outer lobe) }\end{array}$ & $\begin{array}{c}\text { Values } \\
\text { (measured by inner lobe) }\end{array}$ \\
\hline Lobe number & 18 & 18 \\
\hline Outer penetration angle & $19^{\circ}$ & $25^{\circ}$ \\
\hline Inner penetration angle & $31.2^{\circ}$ & $11.6^{\circ}$ \\
\hline Lobe axial length & $0.180 \mathrm{D}$ & $0.254 \mathrm{D}$ \\
\hline Lobe height & $0.170 \mathrm{D}$ & $0.170 \mathrm{D}$ \\
\hline Lobe width & $0.054 \mathrm{D}$ & $0.051 \mathrm{D}$ \\
\hline
\end{tabular}

\subsection{Spatial grid}

Computational domain of the studied model is discretized by the multi-block structured grid consisting of hexahedron elements. Compared with the unstructured grid, the multi-block structured grid is suggested to resolve the complex flows within and downstream the exhaust nozzle with improved precision. For the aim of saving elements, the averaged size of the hexahedron elements covering the ambient domain is bigger than that for the domain within the lobed nozzle. Since the SST $\mathrm{k}-\Omega$ turbulence model is used in the numerical simulations, the first layer of the hexahedron elements is created to satisfy the requirement of $y^{+} \approx 1$. In addition, the growth rate of the element size outward from the wall boundary is 1.25 .

Before performing the investigations concerning the effect of core flow inlet swirl on the aerodynamic performance of the lobed nozzle, a grid test is carried out. The case with a moderate swirl angle of $20^{\circ}$ is selected for the grid test. Four different multi-block structured grids with a gradually augmented number of hexahedron elements are generated, and detailed element numbers for the grids are presented in Tab.2. Through using the computational method as presented in the following section, numerical results for the four grids are acquired. As for the complex flow field within the lobed nozzle, the flow field is characterized by the development of the lobe-induced large-scale stream-wise vortexes. The large-scale stream-wise vortexes are suggested to enhance the mixing process between the core and bypass flow and more energies are dissipated during the forced mixing process. Therefore, two quantities, namely thermal mixing efficiency and total pressure recovery coefficient, are selected for the study of the grid test. Detailed definitions of these two quantities will be presented later. 
Fig.4 shows profiles of thermal mixing efficiency within the mixing region downstream of the lobed discharge plane for all the tested grids. According to the profiles, it can be demonstrated that all the four grids are able to predict the primary development tendency of thermal mixing efficiency, but the magnitudes of thermal mixing efficiency in each cross section are influenced by the gradually increased elements. As for the case 1, magnitudes of thermal mixing efficiency in all the studied cross sections are depicted to be higher than those for the other three cases. A conclusion can be drawn that the case 1 with an element number of 3 million cannot predict the thermal mixing efficiency with acceptable precision. When comparing with case 4 with respect to the thermal mixing efficiency, case 2 is depicted to increase $12.3 \%$ in the $\mathrm{L} / \mathrm{D}=0.05$ cross section and $1.75 \%$ in the last studied cross section, respectively. Based on the presented profiles for the grid 3 and grid 4, no noticeable differences between these two profiles are observed. Profiles of total pressure recovery coefficient for all the tested cases are presented in Fig.5. In all the studied cross sections magnitudes of total pressure recovery coefficient for the case 1 have decreased obviously as compared with the other three tested cases. Specifically, in the last studied cross section the magnitude of total pressure recovery coefficient for the case 1 has decreased $0.4385 \%$ when comparing with the case 4 . Case 2 , case 3 and case 4 nearly predict the same magnitude of total pressure recovery coefficient in all the studied cross sections.

Based on the discussion on both thermal mixing efficiency and total pressure recovery coefficient for all the cases with different element numbers, both the case 3 and case 4 are suggested to predict the two quantities with reasonable precision. Furthermore, in consideration of the limited computational resources, the tested case 3 consisting of 7 million structured elements is thought to be the desired case after the grid test and the mesh of the case 3 is presented in Fig.6.

Tab.2 Element numbers of the multi-block structured grids incorporated in grid test

\begin{tabular}{|c|c|}
\hline Grid case & Total element number \\
\hline Case 1 & 3 Million \\
\hline Case 2 & 5 Million \\
\hline Case 3 & 7 Million \\
\hline Case 4 & 9 Million \\
\hline
\end{tabular}




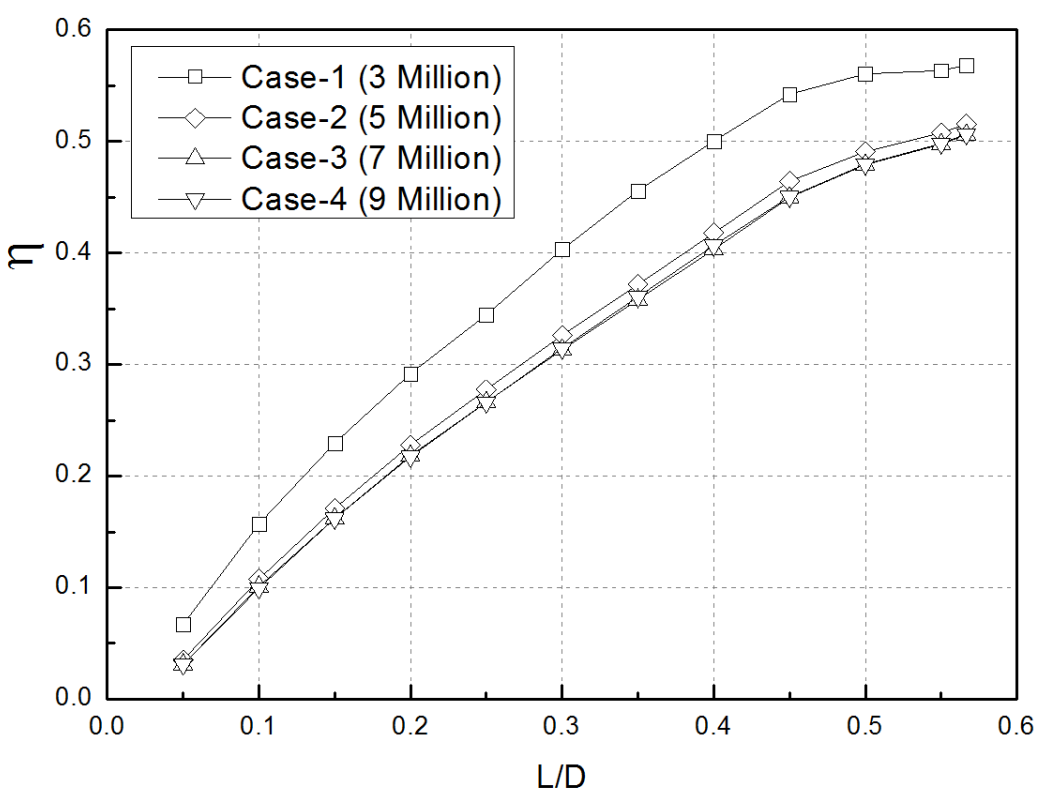

Fig.4 Profiles of thermal mixing efficiency for grid test

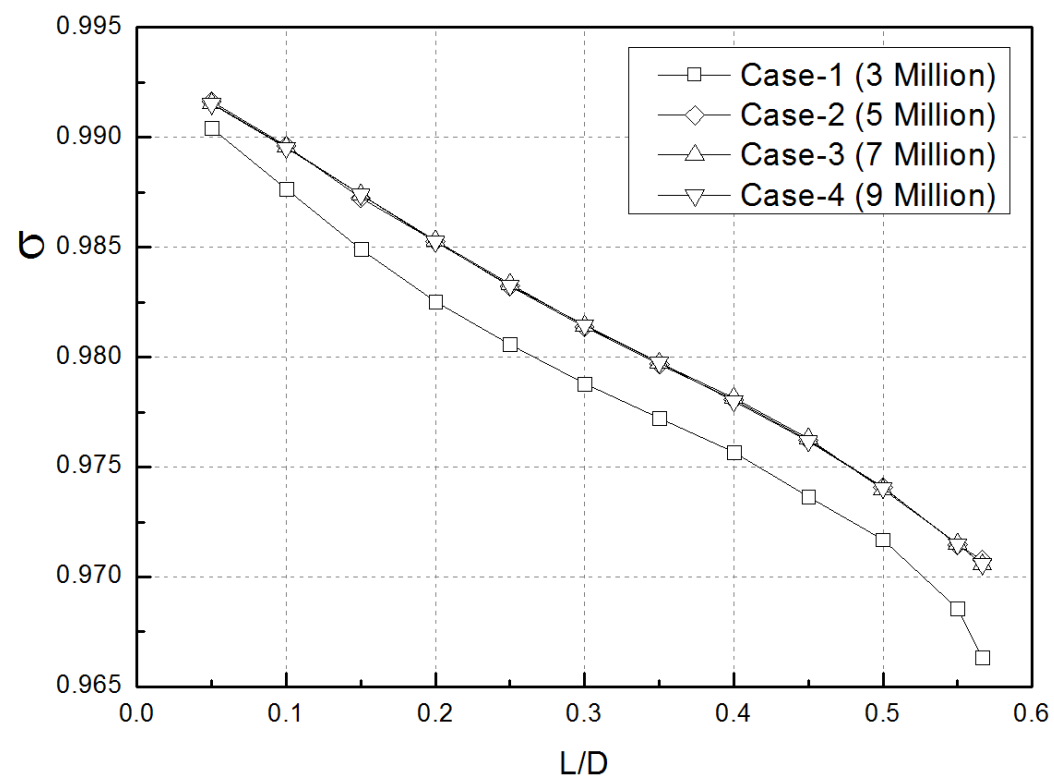

Fig.5 Profiles of total pressure recovery coefficient for grid test
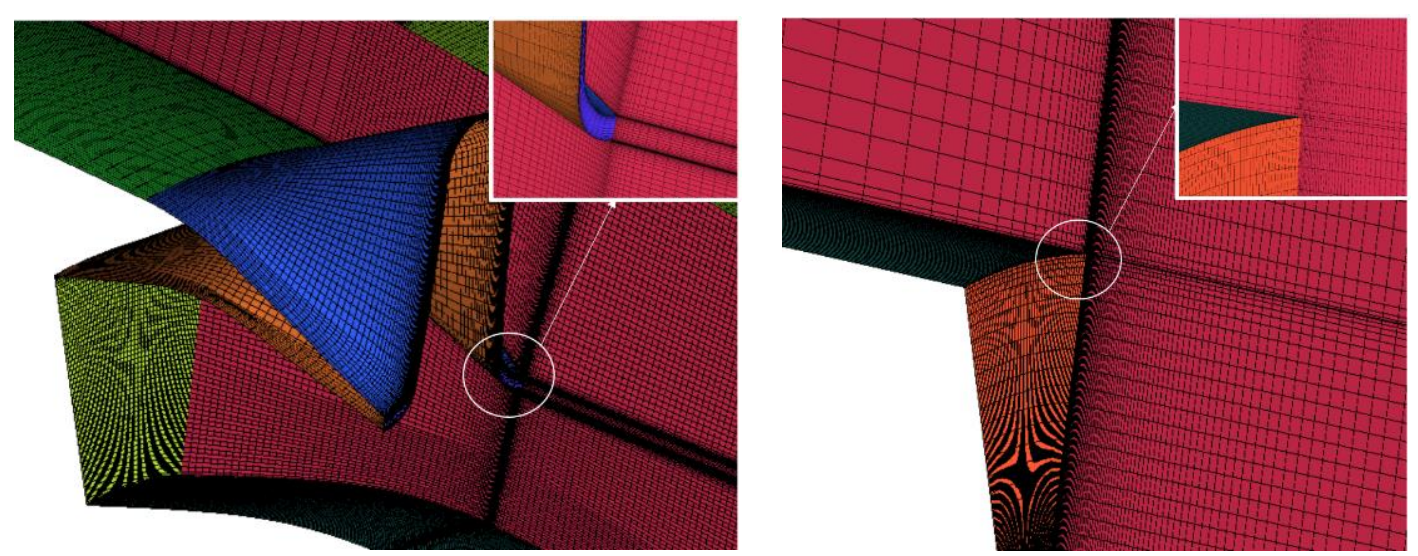
(a) Mesh of the lobe surface

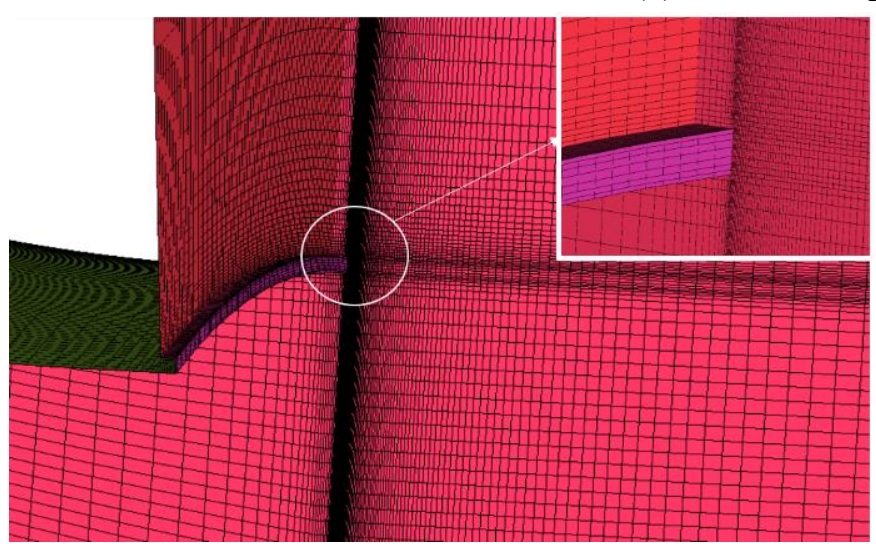

(c) Mesh of the ambient flow entrance

Fig.6 Mesh of investigated geometrical model after grid test

\subsection{Computational method}

All the numerical investigations presented in this paper are conducted by using the commercial Computational Fluid Dynamics (CFD) software Fluent version 14.0 which is thought to be the one with high simulation precision. The complex flow fields within the studied lobed nozzle are obtained by numerically solving the three-dimensional steady RANS equations. The SST $\mathrm{k}-\Omega$ turbulence model is utilized to model the Reynolds stress. Based on the fact that the operating condition used to study the effect of core flow inlet swirl on the performances of the lobed nozzle is a real working state, the averaged Mach number of the exhausting jet is far beyond 0.3. And therefore, the flow is regarded as the compressible flow with the ideal gas law introduced to calculate the density of the compressible flow. Note that the core flow is the real hot gas exhausting from the low-pressure turbine, and the bypass flow is considered as the air flow. The exact concentration of the mixed flow downstream of the scarfed lobed mixer is acquired by solving the discretized species transport equations. In order to obtain simulation results with high precision, the terms incorporated in the equations are all discretized by high-order schemes that the pressure term is discretized by the second order scheme and the other terms are all discretized by the second order upwind scheme. The coupled method in Fluent is used as the algorithm to couple pressure and velocity during the iterating process.

As for the detailed boundary conditions, the core inlet, bypass inlet and ambient flow inlet are set to be the pressure inlet boundary. It is noted that according to the pressure inlet boundary of the ambient flow, a constant Mach number of 0.75 is generated. A zero shear stress condition is specified on the boundary of the ambient flow field, and a pressure outlet boundary is defined at the outlet of the computational domain with a magnitude of the ambient pressure. Detailed aerodynamic parameters specified at these boundaries are presented in Tab.3. Magnitudes of pressure and temperature have been normalized by the ambient pressure and the ambient temperature, respectively. In addition, both the nozzle boundary and the lobe surface are set to be the wall boundary with a smooth, adiabatic and non-slip surface. As aforementioned that only a sector domain corresponding to one lobe body was studied, and thus sidewalls of the studied sector field are set to be the rotational periodic boundaries. In order to simulate swirl motions of the core flow, a constant swirl angle is imposed on the inlet cross section of the core flow with the swirl angle set to be $0^{\circ}, 5^{\circ}, 10^{\circ}, 15^{\circ}, 20^{\circ}, 25^{\circ}$ and $30^{\circ}$, respectively. 
Numerical iteration processes are carried out on a server with two Intel Xeon E5-2640 processors and a physical memory of $64 \mathrm{~GB}$. When performing the iterations by standard wholefield initialization, approximately 50,000 iterations are required before the iteration process reaching a converged result. It is worthy to note that two convergence criterions should be met before the convergence declaration. Firstly, all scaled residuals of the iterating equations should be less than $10^{-5}$. Secondly, the mass flow rate of the nozzle outlet should be constant to five significant digits at least.

\begin{tabular}{|c|c|c|c|}
\hline \multicolumn{4}{|c|}{ Tab.3 Boundary conditions used in the numerical investigations } \\
\hline Properties & Total pressure & Total temperature & Turbulent intensity (\%) \\
\hline Core flow inlet & 1.7514 & 3.3754 & 5 \\
\hline Bypass flow inlet & 1.9411 & 1.2641 & 5 \\
\hline Ambient flow inlet & 1.4522 & 1.1124 & 3 \\
\hline
\end{tabular}

\section{Computational method validation}

Lei [18][19] et al. has conducted a series of experiments to study the influence of core flow inlet swirl on both the forced mixing mechanism and aerodynamic performances of the lobed nozzle. Several fundamental conclusions were drawn depended upon their research results. Therefore, in order to quantitatively evaluate the simulation accuracy, the computational method utilized in this paper is validated against the experimental results published by Lei [20]. Lei has measured decay profiles of the circulation in the cross sections perpendicular to the main flow direction with gradually augmented core flow inlet swirl angles. And the case with the maximum swirl angle of $30^{\circ}$ is simulated by the presented computational method since the maximum swirl angle is thought to induce the most significant swirling effect on both the mixed flow field and the circulation values. It is noted that circulations are considered to characterize circumferential motions of the mixed flow well and thus, quantitative comparisons are conducted between the experimental and simulated solutions. By utilizing the computational method as presented in the last section, the simulation result is obtained and the detailed comparisons with respect to both the counter-clockwise and clockwise circulation are illustrated in Fig.7 and Fig.8, respectively. It is noted that the presented computational method consists of the pressure-based solver and the coupled method. As for the solver in Fluent software, the density-based solver is suggested to predict the complex flow field with enhanced precision sometimes but at the expense of increased computational resources. Thus for the aim of detailed comparison, the simulation results obtained through using the density-based solver are also depicted in Fig.7 and Fig.8.

As for the counter-clockwise circulations by using the pressure-based solver, with respect to the experimental result the magnitude is over predicted with a relative error of $6.01 \%$ in the first compared cross section. And the predicted values are $1.43 \%$ and $0.78 \%$ smaller in the subsequent two cross sections. Through using the computational method with the SST $\mathrm{k}-\Omega$ turbulence model incorporated, the lobe-induced forced mixing process is more pronounced within the flow field slightly downstream of the lobed mixer causing the counter-clockwise circulation with an augmented decay rate as presented in Fig.7. In the fourth cross section, the value measured by the experimental effort has dropped to 0.98 and the corresponding relative prediction error in this cross section for the presented numerical method is $11.9 \%$. Although this prediction error surpasses the averaged value, the value of the counter-clockwise circulation in the fourth cross section has 
decreased markedly, and the influence induced by the counter-clockwise circulation is weakened. Based on the comparison a conclusion can be drawn that the predicted counter-clockwise circulations by using the pressure-based solver are in good agreement with the experimental measurements except small but acceptable disagreements in some studied cross sections. As for the profile in Fig.7 obtained by using the density-based solver the simulation precision is in the same level of the pressure-based solver except for the third cross section. In this cross section the relative prediction error with the density-based solver used is $9.03 \%$ which has increased apparently as compared with the prediction error by using the pressure-based solver.

With regard to the experimentally measured decay profile of the clockwise circulation, the clockwise circulation is depicted to have a sharp decline within the flow field between the first and second cross section. While this rapid decreasing rate is not captured in the numerical simulation by using the pressure-based solver and thus resulting into the over predictions in both the second and third cross section as presented in Fig.8. The simulation results with the pressure-based solver incorporated are $16.1 \%$ and $15.6 \%$ bigger than those of the experimental magnitudes. When the pressure-based solver is replaced by the density-based solver in Fluent the simulation precision is found to be improved. In detail, the relative errors by using the density-based solver are $11.0 \%$ in the second cross section and $5.60 \%$ in the third cross section, respectively.

According to the comparisons between the experimental and numerical results, the decay trend obtained by the numerical method agrees reasonably well with the experiments regarding both the counter-clockwise and clockwise circulation. When comparing with the predictions of the pressurebased solver, the clockwise circulations obtained by the density-based solver are improved but at the expense of increased error for the counter-clockwise circulations and the augmented time required for the iteration process. Therefore, the presented computational method with both pressure-based solver and the SST $\mathrm{k}-\Omega$ turbulence model is suggested to be capable of predicting the circulations with acceptable predicting precision, and the presented computational method can be used to resolve the mixed flow field with the presence of core flow inlet swirl motions.

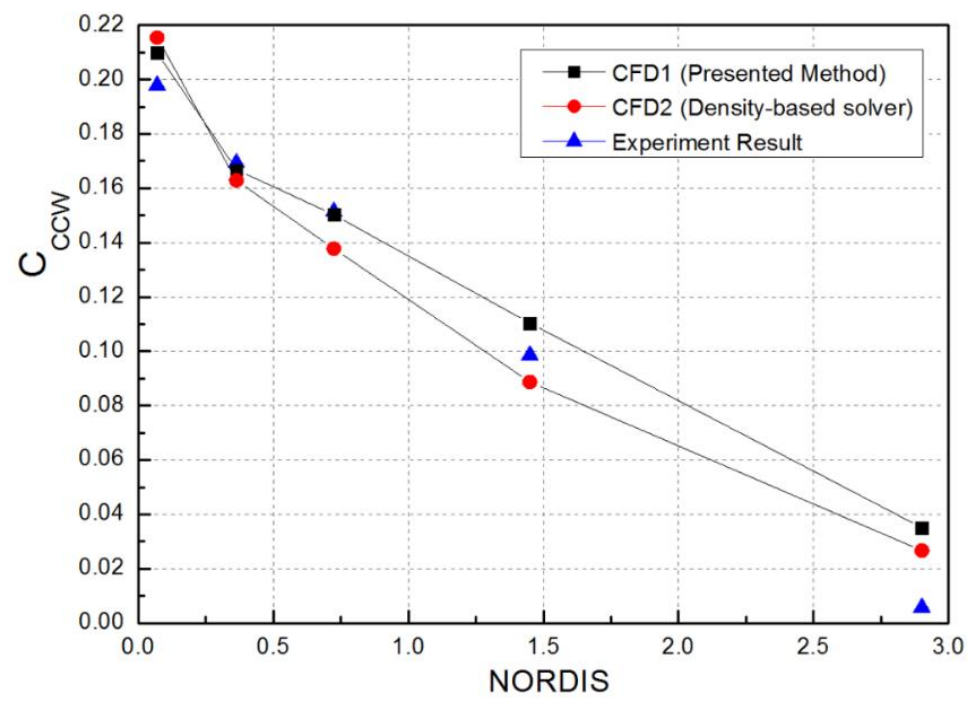

Fig.7 Comparison of normalized counter-clockwise circulations between experiment and CFD 


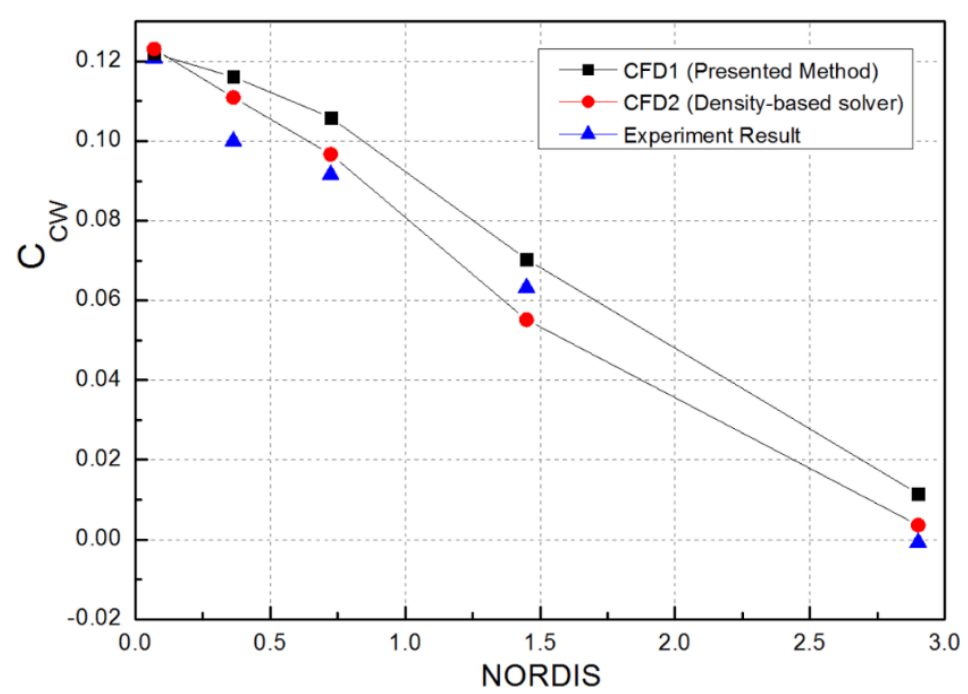

Fig.8 Comparison of normalized clockwise circulations between experiment and CFD

\section{Data Reduction}

The stream-wise vortex is discussed for the mixed flow field downstream of the lobed mixer, and the detailed equation is presented in Eq-1. The axial velocity as incorporated in the following contours have been normalized by the magnitude of the axial velocity in the core inlet cross section for the baseline case. The two co-flows are gradually mixed mainly due to the vortex system and the swirling motions, and thus a quantity named as thermal mixing efficiency is used to evaluate the mixing efficiency of the mixed flow as depicted in Eq-2. And the fundamental quantities in Eq-2 are presented in Eq-3 and Eq-4. Thermal mixing efficiency is first defined and used by Prof. Liu and Dr. Xie [21]. During the mixing process the loss of the fluid energy is inevitable, and the total pressure recovery coefficient is used to determine the loss which is calculated by Eq- 5 .

$$
\begin{aligned}
& \omega_{S T V}=\frac{D}{u_{\text {core }}}\left(\frac{\partial w}{\partial Y}-\frac{\partial v}{\partial Z}\right) \\
& \eta=1-\frac{\int\left(T-T_{c-m i x}\right)^{2} d\left(C_{p} \dot{m}\right)}{D(T)_{c}} \\
& T_{c-\text { mix }}=\frac{T_{c \text { ore }} \dot{m}_{c \text { ore }} C_{p, \text { core }}+T_{\text {fan }} \dot{m}_{\text {fan }} C_{p, \text { fan }}}{\dot{m}_{\text {core }} C_{p, \text { core }}+\dot{m}_{\text {fan }} C_{p, \text { fan }}} \\
& D(T)_{c}=T_{c o r e}^{2} \dot{m}_{c o r e} C_{p, c o r e}+T_{f a n}^{2} \dot{m}_{f a n} C_{p, f a n} \\
& -T_{c-\text { mix }}^{2}\left(C_{p, \text { core }} \dot{m}_{c o r e}+C_{p, f a n} \dot{m}_{f a n}\right) \\
& \sigma=\frac{p_{m i x}^{*}}{p_{i n}^{*}}
\end{aligned}
$$

\section{Results and discussion}

Through using the presented computational method which has been validated against experimental data, mixed flow fields for the investigated cases with gradually augmented core flow inlet swirl angles are obtained and therefore the effect of core flow inlet swirl on aerodynamic performances of the lobed nozzle is quantitatively investigated. This chapter is divided into two major segments: the first segment consists the 5.1 section which is designed to present the characteristics of the flow field for the baseline case. And the second segment comprises the rest portions. During the second segment, the effects of core flow inlet swirl on both the mixed flow 
field and the aerodynamic performances of the lobed nozzle are studied.

\subsection{Flow and mixing characteristics for the baseline case}

Fig. 9 and Fig. 10 present axial velocity contours in the meridional planes at the lobe crest centerline and the lobe valley centerline, respectively. The velocity vectors generated by the axial and radial velocity are also illustrated in this figure. With respect to the axial velocity contour at the lobe crest centerline in Fig.9, a reversed flow zone can be figured out at the very start point of the lobe expansion section. Due to the fact that the lobe crest with a large penetration angle is used in this study, the boundary layer separation of the core flow takes place at the starting point of the lobe expansion section, and thus the reversed flow zone is formed. By the end of the lobe crest, a small fraction of core flow with low axial momentum gathers in the vicinity of the lobe crest. As soon as the core fluid flows to the tip point of lobe crest, flow direction of the core flow is forced to deflect to the bypass fluid side. Within the deflection process, the deflected core flow with low axial momentum is accelerated by the high-speed bypass flow through viscous shear stress, resulting into the S-shaped streamlines near the tip point of lobe crest. For the two contours in different meridional planes in Fig.9 and Fig.10, two large-scale reversed flow zones induced by the tip point of the plug body are observed, and the fluid with low axial momentum in the form of a thin strip is depicted to follow the large-scale reversed zones. It is noted that the exhaust nozzle investigated in this study is a converged nozzle, namely the cross section area of the exhaust nozzle is gradually decreased as a response to the enhancement of mixing distance. Consequently, districts with apparently increased axial velocity in the outlet region can be observed which have been marked with the colored ellipses in Fig.9 and Fig.10.

Within the mixed flow field downstream of the lobed mixer, the large-scale stream-wise vortex is indicated to play a major role in predicting the mixed flow field. Detailed contours of these vortexes at five studied cross sections are presented in Fig.11. Due to the inner and outer penetration body of the lobed mixer, pairs of counter-rotating stream-wise vortexes are induced in the subsequent flow field. And the radial length scale of the induced stream-wise vortexes is on the order of the height of the studied lobed mixer as shown in the depicted cross section with $\mathrm{L} / \mathrm{D}=0.05$. As for the contours of the normalized stream-wise vortex field with $\mathrm{L} / \mathrm{D}=0.05$ and 0.1 , it can be seen that the district influenced by the large-scale stream-wise vortex around the lobe valley is bigger than that for the large-scale stream-wise vortex around the lobe crest. Because of the lobe scarfing optimization, the large-scale stream-wise vortex pairs induced around the lobe valley are ahead of the stream-wise vortex pairs around the lobe crest, and therefore the vortex pairs around the lobe valley are more pronounced in the cross sections with $\mathrm{L} / \mathrm{D}=0.05$ and 0.1 . Although the stream-wise vortex pairs around the lobe valley are more pronounced in the cross sections upstream of $\mathrm{L} / \mathrm{D}=0.1$, the dissipation process of the stream-wise vortex pairs is suggested to be much faster which is confirmed by the fact that no stream-wise vortex pairs around the lobe valley can be observed downstream of the $\mathrm{L} / \mathrm{D}=0.2$ cross section. At the $\mathrm{L} / \mathrm{D}=0.05$ cross section, the stream-wise vortex pair induced by the lobe crest has the maximum magnitude. As the stream-wise vortex pair around the lobe crest moving downstream, more bypass fluids are entrained into the vortexes, and the large-scale stream-wise vortex is gradually diffused. It can be inferred that the uniformity of the mixed core and bypass fluid around the lobe crest is augmented by the entrainment process. For all the studied cross sections, the strength of the stream-wise vortex pair around the lobe crest is shown to be reduced as a result of the enhanced mixing distance. In the last studied cross section with 
$\mathrm{L} / \mathrm{D}=0.55$, the strength of the stream-wise vortex pair around the lobe crest has diminished apparently and the magnitude of the stream-wise vortex pair around the lobe crest is on the order of 30 .

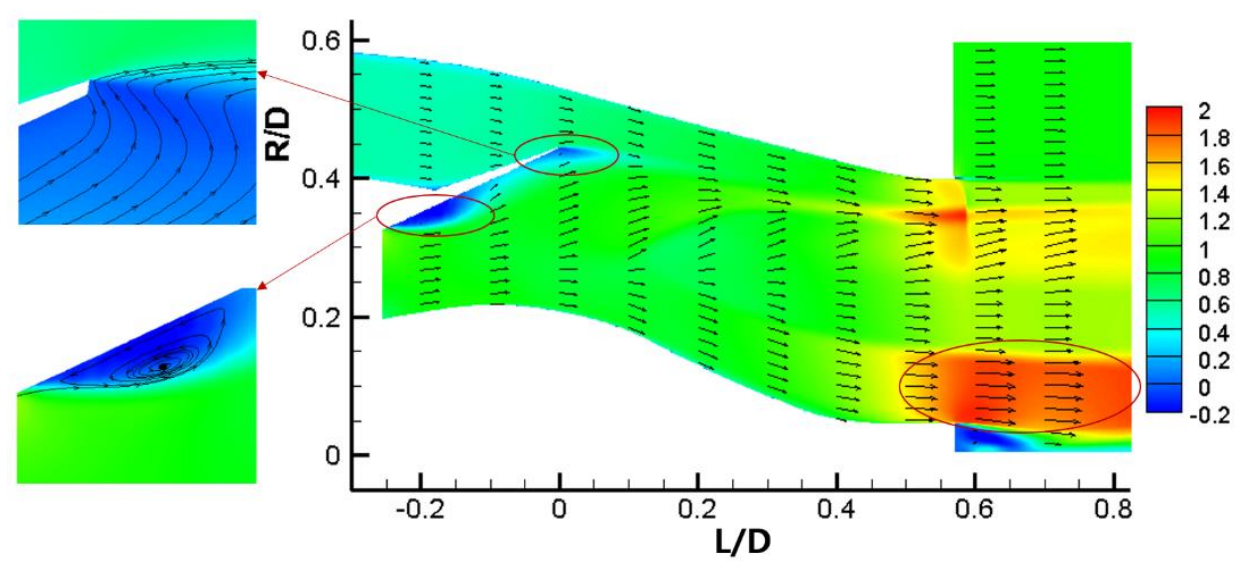

Fig.9 Contour of axial velocity in the meridional plane of the lobe crest for the baseline case

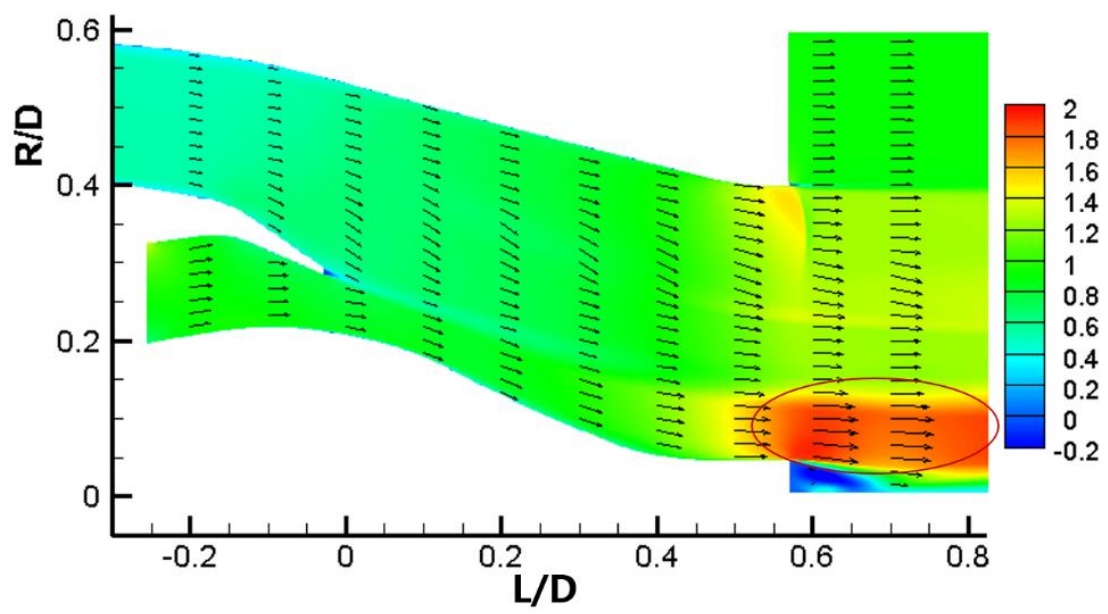

Fig.10 Contour of axial velocity in the meridional plane of the lobe valley the baseline case

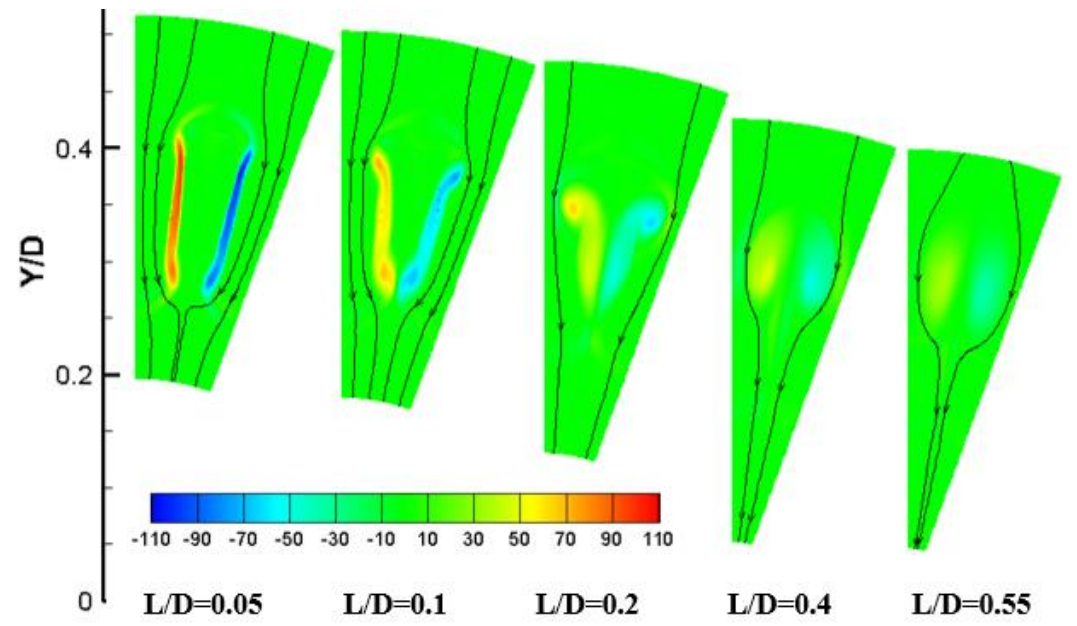


Fig.11 Contours of stream-wise vortexes in the cross sections for the baseline case

\subsection{Influence of Inlet Swirl on Mixed Flow Field}

With enhanced swirling motions of the core flow, the mixed flow field can be greatly affected, and the impacts are discussed in this section. Normalized axial velocity contours in the meridional planes at the lobe crest centerline for the $10^{\circ}$ and $30^{\circ}$ case are presented in Fig. 12 and Fig.13, respectively. As for the meridional plane at the lobe crest, a reversed flow zone at the initial expansion section of the lobe crest body can be observed for the $10^{\circ}$ case which can also be obtained for the baseline case as already studied in the 5.1 section. Compared with the reversed flow zone for the baseline case, the size of the reversed flow zone for the $10^{\circ}$ case is shown to have decreased a lot, and only a small reversed flow zone can be figured out for the $30^{\circ}$ case in the meridional plane near the lobe crest body.

For the aim of discussing the mechanism behind this gradually dissipated reversed flow zone, axial shear stress contours of the core flow surface of the lobed mixer for four cases are depicted in Fig.14. Surface axial shear stress is directly influenced by the axial velocity gradient in the direction normal to the surface, and the start-point of the reversed flow emerges as soon as the axial shear stress decreases to zero. As presented in the studied cases with a swirling angle of $0^{\circ}, 10^{\circ}$ and $20^{\circ}$, both the area and strength of the negative surface shear stress are shown to be enhanced as a response to the gradually increased swirling angle. This development process indicates that the flow field influenced by the reversed flow is enlarged, and the reversed flow zone induced by the expansion section of the lobe crest body is more pronounced. At the condition of augmented swirling angle (but still less than $20^{\circ}$ ), circumferential motions of the core flow are strengthened and more flow field in the vicinity of the lobe crest is affected by the reversed flow zone due to the viscous shear stress between the fluid elements. And therefore, the enlarged reversed flow region shows up. At the cases with a swirling angle greater than $20^{\circ}$, both the area and strength of the negative surface shear stress are alleviated as a result of the enhanced core swirling flow which is contrary to the obtained observations for the swirling cases having a swirling angle less than $20^{\circ}$. For the highly swirling cases the separated flow is suggested to receive energies from the high circumferential motions to overcome the adverse pressure gradient in the expansion section of the lobed mixer, leading to both the decreased strength and reduced area of the negative surface axial shear stress. Furthermore, a small reversed flow zone at the condition of $30^{\circ}$ in the meridional plane near the lobe crest body was observed in Fig. 14. When the swirling angle is increased from $20^{\circ}$ to $30^{\circ}$, the district of the axial surface shear stress with a negative value is broken into two discrete slender strips located in the initial expansion section of the lobed mixer as depicted in Fig.14. One of the slim strips marked with STRIP1 is formed by the main negative district as shown in the $20^{\circ}$ case. The other strip marked with STRIP2 is also due to the breaking process but with enhanced circumferential motions, the generated STRIP2 is transferred circumferentially downstream, and therefore the STRIP2 located on the right side of the STRIP1 with the periodic boundary condition used in the numerical simulation process. According to the presented four contours in Fig.14, a nearly triangle-shaped negative region can be obtained in the region approximating the lobe crest outlet cross section as marked with a colored ellipse in the baseline case. Nevertheless, the negative region dissipates in all the swirling cases which indicates that core swirling flow can accelerate the low axial-momentum fluid near the outlet cross section of the lobed mixer and therefore prevent the axial reversed flow zone near the outlet tip-point of lobe crest. 
Core swirling flow is induced at the entrance of the core flow and then travels downstream with the main core fluid. Compared with the core swirling flow between the lobe valley and the plug body, the core swirling flow existing in the flow field between the lobe valley and crest is significantly influenced by the lobe body. Meanwhile, the developed core swirling flow between the lobe valley and crest is suggested to affect the mixing flow field downstream of the lobe trailing edge. Therefore, streamlines of the flow field with $\mathrm{L} / \mathrm{D}=-0.05$ for the studied cases with a swirling angle of $0^{\circ}, 10^{\circ}, 20^{\circ}$ and $30^{\circ}$ are presented in Fig. 15, respectively. The streamlines depicted in Fig. 15 are generated by the defined velocity vector which is the combination of radial velocity and circumferential velocity. Each lobe body of the mixer can be regarded as an axial blade with $0^{\circ}$ angle and thus, two side walls of the lobe body act as the pressure surface and the suction surface of the blade. At the baseline case, there is no swirling flow at the core entrance cross section and the flow fields in the vicinity of each lobe side wall are suggested to be the same which leads to the conclusion that the pressure surface is indistinguishable from the suction surface for the baseline case. For the swirling cases in Fig.15 swirling direction of the core flow is from right to left and thus the left side wall of the depicted lobe body is thought to act as the pressure surface of a blade, at the same time the right side wall serves as the suction surface. When the core swirling angle is gradually increased, the difference between the pressure surface and the suction surface is depicted to be more obvious. For the $10^{\circ}$ case, a reversed flow zone is obtained on the suction surface which is not figured out in the baseline case. In the swirling case, an adverse pressure gradient occurs on the lobe suction surface, and the reversed flow zone takes place after the separation point which is located on the suction surface a few distance downstream of the leading edge. As soon as the core swirling angle is increased to $20^{\circ}$, the observed reversed flow zone as shown at the $10^{\circ}$ case has enlarged. It is noted that as depicted in the figures vortex cores of the reversed flow zone for both the $10^{\circ}$ and $20^{\circ}$ case are shown to be approximately located in the same place. When the swirling angle is augmented further to $30^{\circ}$, the separation point on the suction surface is put forward to the position more close to the leading edge of the lobe body. In addition, the whole one reversed flow zone as shown in the $10^{\circ}$ case and the $20^{\circ}$ case is broken into two slender reversed flow zones at the condition of $30^{\circ}$ core swirling angle.

As already studied by many researchers [22][23] that the key mechanism concerning the outweighed mixing performance of the lobed mixer is two counter-rotating large-scale stream-wise vortexes downstream of the lobed trailing edge. Therefore, the reversed flow zone on the lobe suction surface induced by the swirling flow is suggested to interact with the stream-wise vortexes in the subsequent mixing flow field causing the stream-wise vortexes to deform and transfer. Another phenomenon depicted in Fig. 15 is the transportation of the stagnation point. A stagnation point caused by the streamlines originating from different parts of the lobed mixer body is formed in the presented cross section and the stagnation points for all the depicted cases have been marked with STAP. During the process of increasing the swirling angle, the stagnation point gradually deviates from the centerline of the lobe crest and heads for the pressure surface. Under the condition with strengthened core swirling motions, the stagnation point is pushed downstream in the swirling direction through increased circumferential momentum which results in the shortened distance between the lobe pressure surface and the stagnation point.

Fig. 16 shows the axial velocity contours of the $10^{\circ}$ case in five cross sections downstream of the lobed trailing edge plane. The velocity vectors obtained by the radial and circumferential velocity are also depicted in Fig.16. Since the area of the lobed nozzle is gradually decreased, the 
averaged axial velocity of the mixed flow is experiencing an accelerating process. By referring to these contours the averaged axial velocity of the mixed flow in the $\mathrm{L} / \mathrm{D}=0.05$ cross section is 0.7 around which has enhanced to 1.4 around in the $\mathrm{L} / \mathrm{D}=0.55$ cross section. A high-speed region with a magnitude of 0.9 around can be figured in the $L / D=0.05$ cross section and this region is nearly covered by the boundary of the lobe body. During the forced mixing process downstream the highspeed region is influenced by both the stream-wise vortexes and the swirling motions. With the extended mixing distance the high-speed region is narrowed and forced to travel from the core flow district to the bypass district approaching the top wall boundary of the exhaust nozzle. In the last studied cross section with $\mathrm{L} / \mathrm{D}=0.55$ the high-speed region is nearly shaped into a circle region and the high-speed area is characterized by the magnitude of 1.9 around. For the region between the lobe valley and the plug surface, the flow field development is less affected by the lobe-induced large scale stream-wise vortexes and but predominated by both the swirling motions and the shape of the plug body. Within the subsequent flow field of the lobed mixer, the radius of the plug body is reducing. For the angular momentum balance of the flow near the plug body, the swirling angle of the fluid is augmented at the presence of the reducing radius of the plug body. With regard to the depicted velocity vector in the $\mathrm{L} / \mathrm{D}=0.55$ cross section, within the flow field near the plug body the radial flow motions are shown to be ignorable as compared with the swirling motions and thus the swirling motions are predominant over this region.

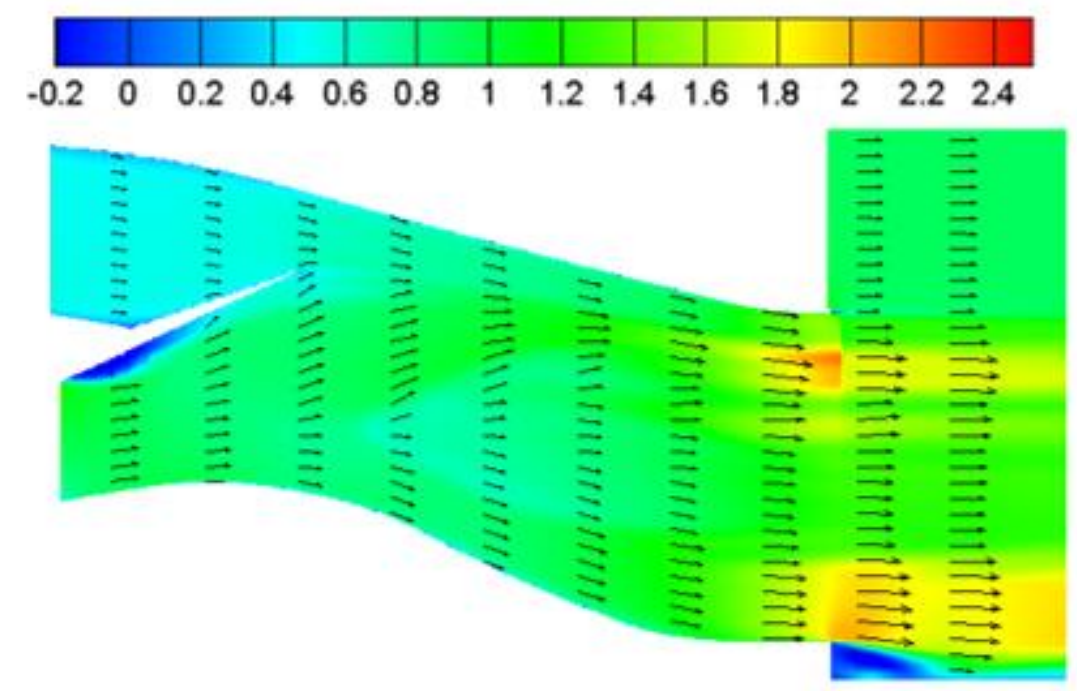

Fig. 12 Contour of axial velocity in the meridional plane of lobe crest for the $10^{\circ}$ case 

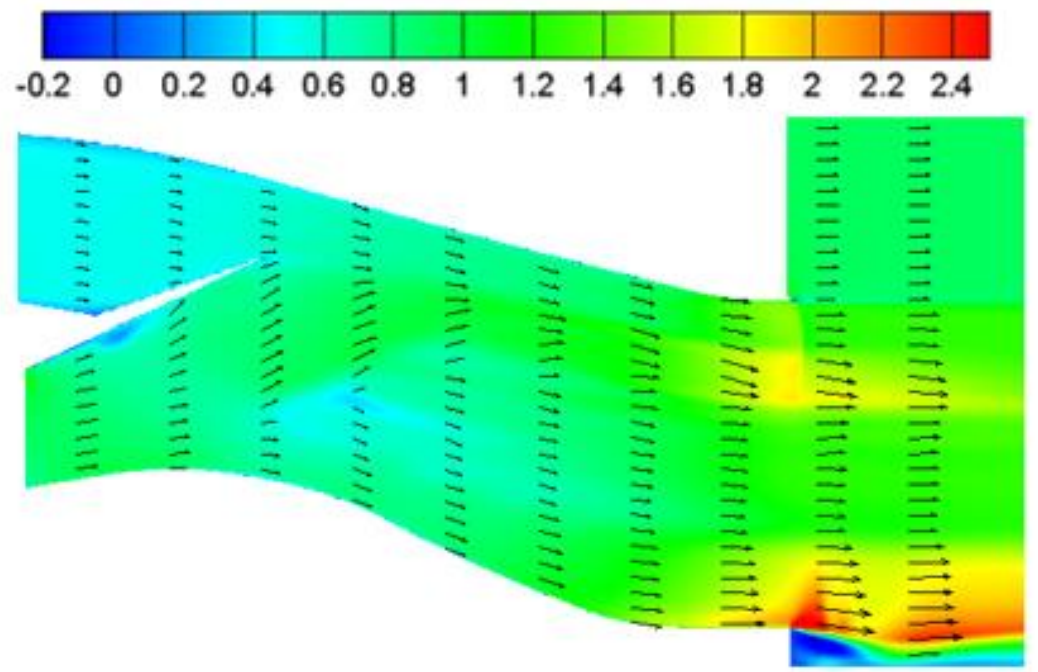

Fig. 13 Contour of axial velocity in the meridional plane of lobe crest for the $30^{\circ}$ case

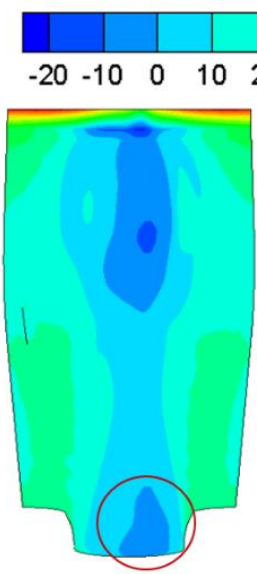

Baseline case

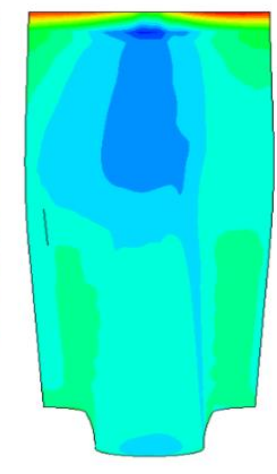

$10^{\circ}$ case

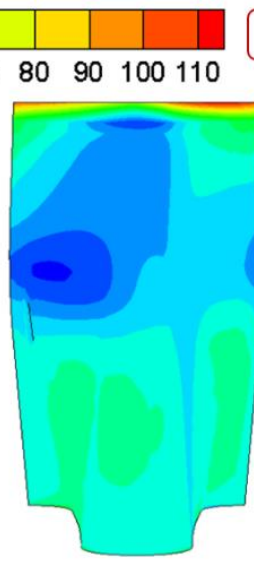

$20^{\circ}$ case

\section{STRIP1 STRIP2}

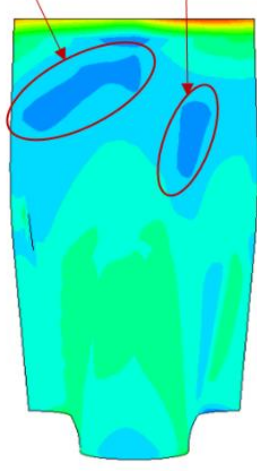

$30^{\circ}$ case

Fig.14 Contours of axial shear stress of the core lobe surface

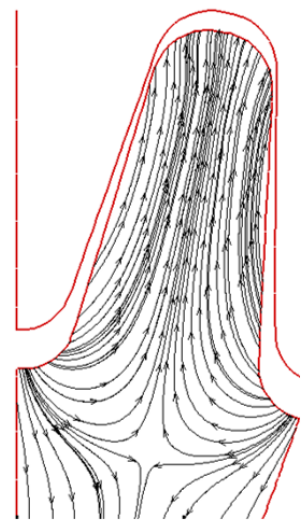

Baseline case

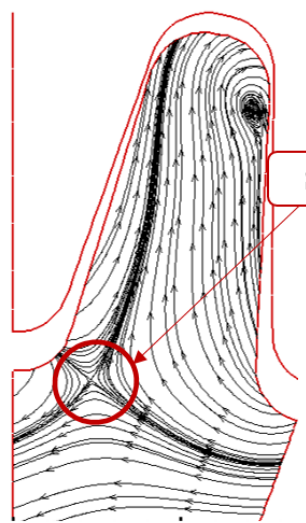

$10^{\circ}$ case

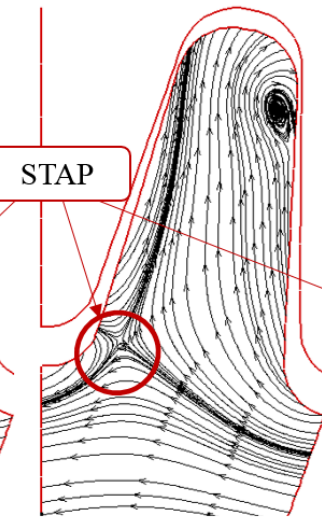

$20^{\circ}$ case

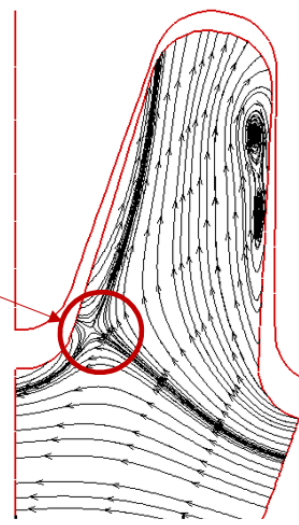

$30^{\circ}$ case

Fig. 15 Streamlines within the $L / D=-0.05$ cross section of the core flow field 


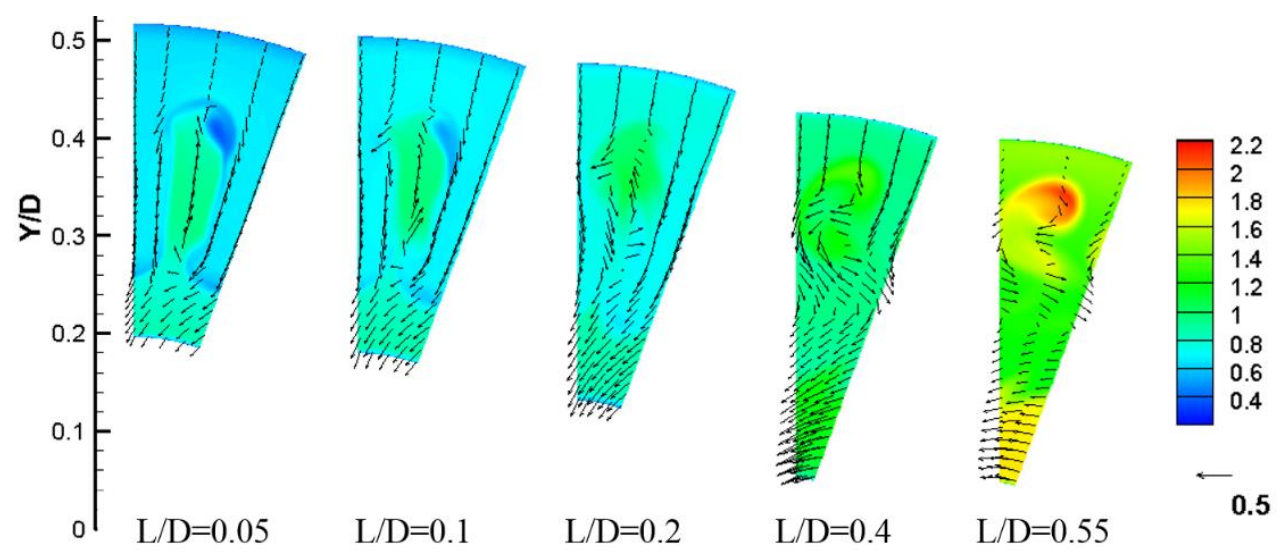

Fig. 16 Contours of axial velocity for the $10^{\circ}$ case

\subsection{Influence of inlet swirl on stream-wise vortexes}

The lobe-induced large scale stream-wise vortexes are regarded as the primary mechanism underlying the mixing process within the lobed nozzle. With the presence of the swirling motions caused by the core flow inlet swirl, the development of the stream-wise vortex is suggested to be influenced by the swirling motions and hence the detailed mixing process downstream would not follow the same pattern as the baseline case. For the purpose of studying the influence of the inlet swirl on the vortex development, contours of stream-wise vortexes in the studied cross sections for the baseline case and two swirling cases are examined. In addition, streamlines in these cross sections are also surveyed since the stream-wise vortex is induced by the flows with opposite directions of radial velocity. Streamlines and contours of stream-wise vortexes are presented in Fig. 17 and Fig.18, respectively. For each of the presented cross sections, it is divided into three parts along the radial direction for the convenience of discussion. The major part is the region dominated by the large-scale stream-wise vortexes and this major part is named as the middle part. Based on the definition of the middle part, the district between the nozzle wall boundary and the middle part is known as the top part and the remaining district is called the bottom part. Within the cross sections slightly downstream of the lobed trailing edge, such as the $\mathrm{L} / \mathrm{D}=0.05$ and 0.1 cross section, the boundaries formed by these three parts can be identified easily. As for the other examined cross sections, on account of both the vortex development and the influence caused by the swirling motions, the boundaries are found to be indistinct. The bottom part is mainly dominated by the swirling motions and the effect of the swirling motions is gradually transferred to the middle part. Resultantly, when the mixing distance is increased, the influence of the swirling motions in the middle part is strengthened and is suggested to cause the boundaries to dim.

In the $\mathrm{L} / \mathrm{D}=0.05$ cross section, the lobe induced stream-wise vortexes of the presented cases are shown to have similar shapes. In the plane of the lobed trailing edge, the core and bypass flow have opposite directions of the radial velocity and thus the stream-wise vortexes are formed. Since the cross section with $\mathrm{L} / \mathrm{D}=0.05$ is just slightly downstream of the lobed mixer, the large-scale stream-wise vortexes have not experienced a fast developing process. Consequently the stream-wise vortexes in the $\mathrm{L} / \mathrm{D}=0.05$ cross section are found to maintain the similar appearance of the lobed trailing edge. When the core fluid flows through the lobed mixer, the majority of the swirling motions are removed by the lobe body, resulting in the stream-wise vortexes having the nearly identical maximum magnitude in the $\mathrm{L} / \mathrm{D}=0.05$ cross section. According to the simulated results maximum magnitudes of the stream-wise vortexes in the $\mathrm{L} / \mathrm{D}=0.05$ cross section for the baseline 
case, $10^{\circ}$ case and $30^{\circ}$ case are $100.60,103.78$ and 105.01 , respectively. As compared with the baseline case, maximum magnitude of the stream-wise vortex has increased $3.16 \%$ for the $10^{\circ}$ case and $4.38 \%$ for the $30^{\circ}$ case. Another meaningful phenomenon within the $\mathrm{L} / \mathrm{D}=0.05$ cross section should be discussed is that as a response to the augmentation of the core flow swirling angle the streamlines within the bottom part are gradually forced to the circumferential direction as illustrated in Fig.17. For the core fluid within the bottom part, the guidance effect of the lobe body is small and the circumferential motions are more pronounced at the condition of boosted swirling angle. Therefore, the directions of the streamlines are gradually changed from the radial direction to the circumferential direction when the core flow with a bigger swirling angle is surveyed.

Through comparing the top parts of the three presented contours with $\mathrm{L} / \mathrm{D}=0.1$ in Fig.18, the distributions are in the similar shape and a conclusion can be drew that core swirling motions have ignorable influence on the flow field of the top part. As for the stream-wise vortexes located in the middle part, the vortex with a positive magnitude is regarded as the PSTV and NSTV for the negative stream-wise vortex. In the $\mathrm{L} / \mathrm{D}=0.1$ cross section, the stream-wise vortexes have already experienced a fast developing process in the upstream flow field. Within the middle part, the PSTV is less influenced by the swirling motions as compared with the NSTV. The swirling direction is along the circumferential direction from the right side to the left side and thus the NSTV is suggested to be ahead of the PSTV with regard to the swirling direction. Because of the drag effect induced by the swirling motions, the NSTV is stretched into several vortexes with smaller scales. And one of the smaller vortexes is formed near the boundary of the middle and bottom part as presented in the $L / D=0.1$ cross section in Fig. 17. The induced smaller vortex is significantly influenced and strengthened by the swirling motions. Meanwhile, the turbulence kinetic energy due to the turbulence motions of the induced vortex is enhanced which results in a fast decay rate of the smaller vortex. As illustrated in the cross sections downstream of the $\mathrm{L} / \mathrm{D}=0.1$ cross section in Fig. 17 and Fig.18, the induced smaller vortex can hardly be figured out and its absolute magnitude is less than 35.57 for the $10^{\circ}$ case and 38.67 for the $30^{\circ}$ case.

For the cross sections downstream of the $\mathrm{L} / \mathrm{D}=0.1$ cross section, the swirling motions within the bottom part are transferring to the middle part through convective and diffusive flow. Furthermore, by comparing the streamlines in $\mathrm{L} / \mathrm{D}=0.2,0.4$ and 0.55 cross section, when the swirling angle is accelerated the streamlines in the vicinity of the bottom part are more dragged by the swirling motions. It is noted that both the PSTV and the NSTV in the $\mathrm{L} / \mathrm{D}=0.05$ cross section for the swirling cases are gradually narrowed down and transferred from the middle part to the top part when the mixing distance is increased. During the transferring process both the strength and the vortex range of the stream-wise vortexes are increased for the case with a bigger core flow swirling angle. Based on the simulation results, absolute magnitudes of the NSTV located in the top part of the $\mathrm{L} / \mathrm{D}=0.55$ cross section is 55.07 for the $30^{\circ}$ case and the magnitude has improved $80.55 \%$ as compared with the $10^{\circ}$ case. 


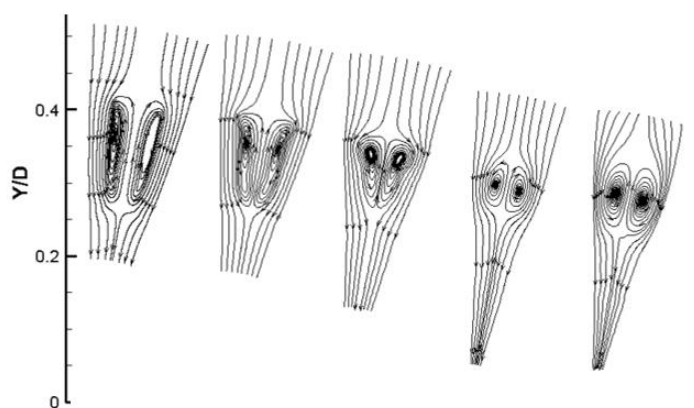

(a) Baseline case
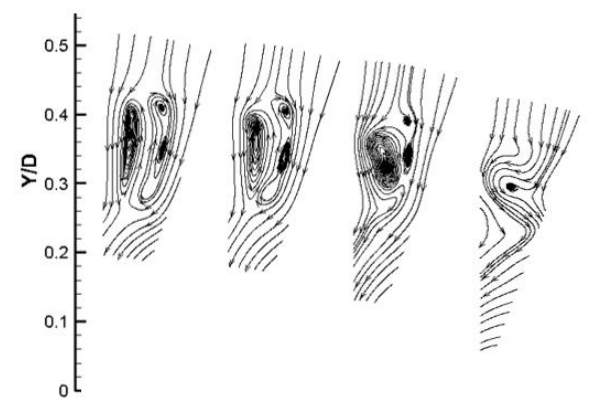

(b) $10^{\circ}$ case
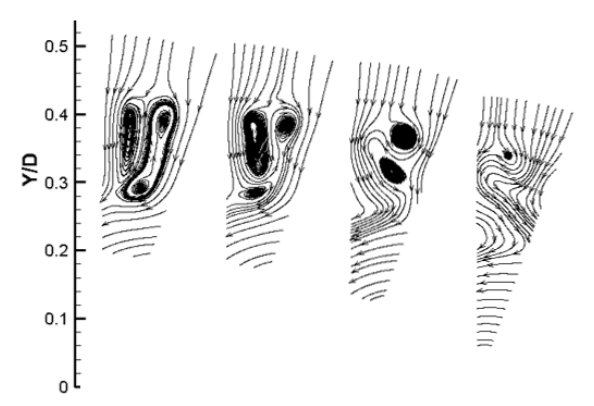

(c) $30^{\circ}$ case

Fig.17 Streamlines in the cross sections for the studied cases

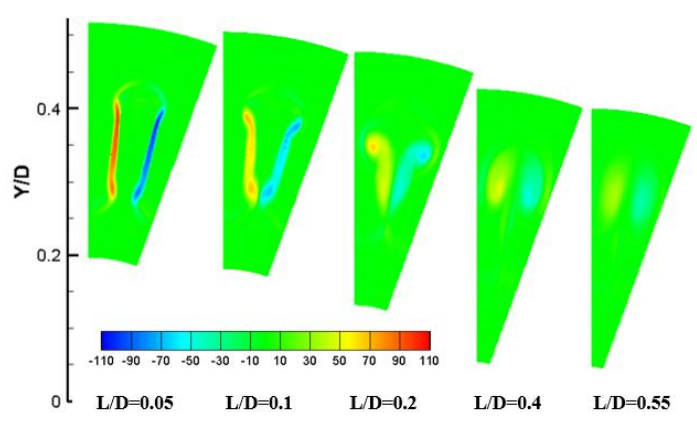

(a) Baseline case

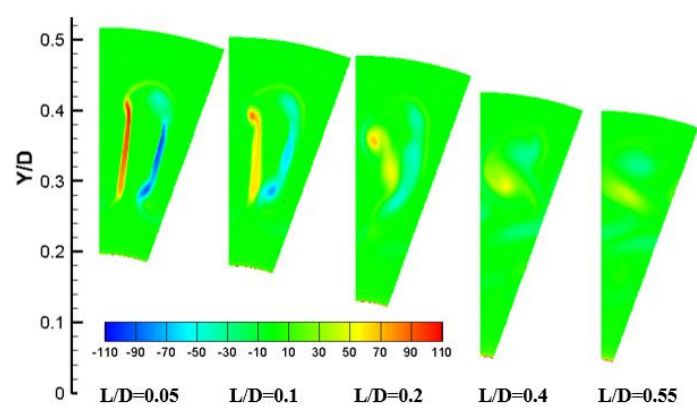

(b) $10^{\circ}$ case

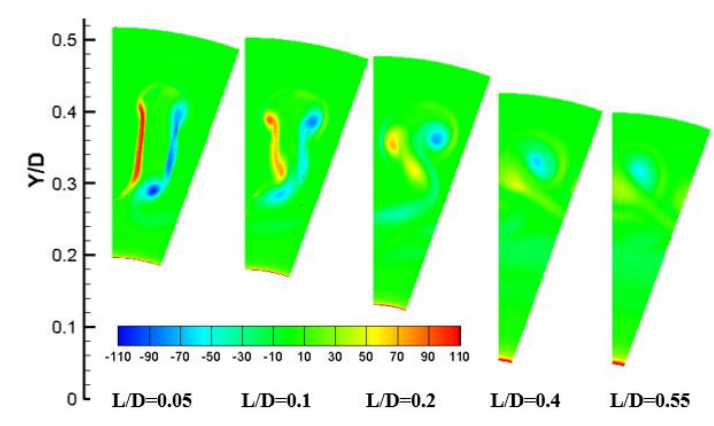

(c) $30^{\circ}$ case

Fig. 18 Contours of stream-wise vortexes in the cross sections for the studied cases

\subsection{Influence of inlet swirl on mixing efficiency and total pressure loss}

Thermal mixing efficiencies of the mixed flow for both the baseline case and the swirling cases are all illustrated in Fig.19. The profiles of the studied cases are obviously influenced by the swirling angle of core flow. As for the mixing flow field between the $\mathrm{L} / \mathrm{D}=0.05$ and 0.45 cross section, the thermal mixing efficiency is nearly gradually enhanced when the swirling angle is increased. In the cross sections downstream of the $\mathrm{L} / \mathrm{D}=0.45$, the discrepancies of thermal mixing efficiency among these swirling cases have been weakened. According to the profiles of thermal mixing efficiency in Fig. 19 , the $15^{\circ}$ case has a profile which almost follows the profile of the baseline case. At the presence of swirling motions, the vortex pattern in the subsequent flow field is negatively influenced in terms of the mixing efficiency. While both the strength and the vortex range of the NSTV are improved for the case with a bigger swirling angle which are suggested to augment the mixing efficiency of the mixed flow. As a consequence, as for the $15^{\circ}$ swirling case the loss caused by the negatively influenced vortex pattern and the benefit induced by the enhanced smaller-scale streamwise vortexes are indicated to almost counteract with each other with respect to the thermal mixing 
efficiency. When the case with a swirling angle less than $15^{\circ}$ is studied, the negatively influence of the vortex pattern is dominating and thus thermal mixing efficiency of the case is reduced. For the $20^{\circ}, 25^{\circ}$ and $30^{\circ}$ case, both the strength and the vortex range of the smaller-scale stream-wise vortexes are more boosted which result in the overall enhancement of thermal mixing efficiency of the mixed flow. In the last studied cross section with $\mathrm{L} / \mathrm{D}=0.566$, the thermal mixing efficiency of the baseline case has a magnitude of 0.477 . The $5^{\circ}$ case is the case with the minimum magnitude of thermal mixing efficiency and the $25^{\circ}$ case for the maximum magnitude in this cross section. As compared with the baseline case, thermal mixing efficiency of the $5^{\circ}$ and $25^{\circ}$ case have decreased $3.15 \%$ and increased $7.73 \%$, respectively.

During the mixing process, the mixing efficiency is gradually enhanced, but the energy dissipation due to the mixing process is also increased. The total pressure recovery coefficient is used for the quantifying of the total pressure loss and the decay profiles of total pressure recovery coefficient for all the studied cases are depicted in Fig.20. In all the studied cross sections the magnitude of total pressure recovery coefficient is shown to be decreased as a response to the augmented swirling angle. When the swirling angle is increased the mixing between the two coflows is strengthened. Therefore, more energies are dissipated during the mixing process. And for the mixing region upstream of the $\mathrm{L} / \mathrm{D}=0.3$ cross section, the discrepancies among these swirling cases are gradually increased. With regard to the mixing region downstream of the $\mathrm{L} / \mathrm{D}=0.3$ cross section the differences are illustrated to be constant. As discussed above the thermal mixing efficiency of the baseline case is as high as the $15^{\circ}$ case. However the difference between the profiles of the baseline case and the $5^{\circ}$ case with respect to the total pressure recovery coefficient is ignorable as depicted in Fig.20. According to the presented profiles in Fig.20, the decreasing rate of the cases is suggested to be nearly linear across the whole studied mixing region. The nearly linear decreasing rate is mainly caused by the short mixing distance downstream of the lobed mixer that the streamwise vortexes, as well as the swirling motions, still exist within the last studied cross section. In the last cross section, both the baseline case and the $5^{\circ}$ case have the same value of total pressure recovery coefficient that is 0.9730 . The $30^{\circ}$ case is depicted to be the case with the minimum total pressure recovery coefficient which has decreased $0.42 \%$ as compared with the baseline case.

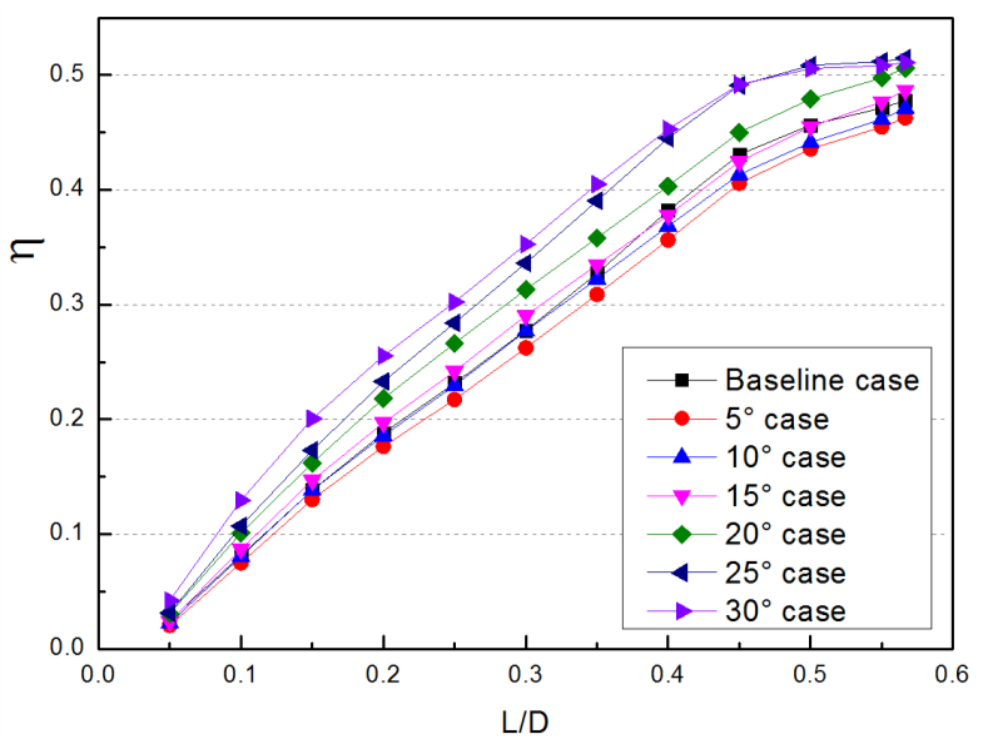


Fig.19 Thermal mixing efficiency for all the studied cases

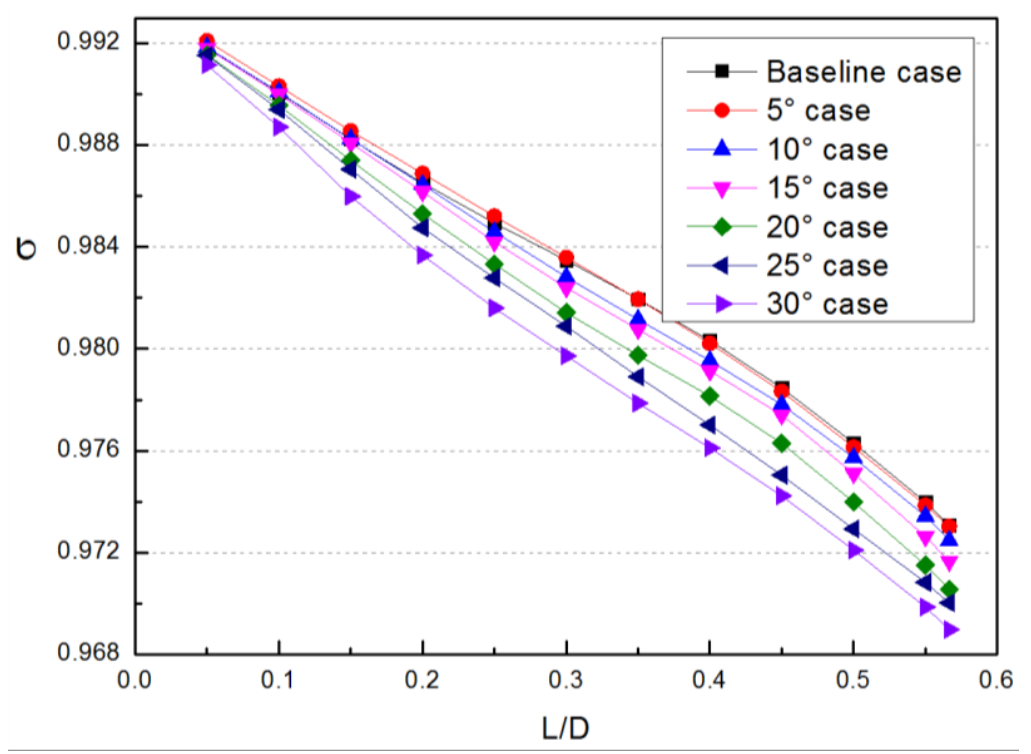

Fig.20 Total pressure recovery coefficient for all the studied cases

\section{Conclusion}

Through solving the N-S equations with the SST $\mathrm{k}-\Omega$ turbulence model, flow fields of the lobed nozzle with increasing core flow inlet swirl angle are acquired. As for the discretization scheme, the pressure term is discretized by the second order scheme and the other terms are all discretized by the second order upwind scheme. And the coupled method in Fluent software is selected as the algorithm to couple pressure and velocity during the iterating process. The presented detailed numerical method which is used to predict to the complex flow field has been validated against experimental measurements, and quantitative comparisons suggest that the prediction precision by using the presented numerical method is acceptable. A series of cases with gradually augmented inlet swirl angle of core flow is studied and the influence of core flow inlet swirl angle on aerodynamic performances of a lobed nozzle is investigated based on the simulation results. The influences of swirling motions on the vortex system, as well as the mixing efficiency and the total pressure loss, are discussed in this paper.

Based on the acquired simulation results, it can be concluded that core flow inlet swirl angle has a great impact on the aerodynamic performances of the lobed nozzle. As for the flow within the middle part, the majority of the swirling motions are removed by the lobe body, resulting in the stream-wise vortexes having the nearly identical maximum magnitude in the $\mathrm{L} / \mathrm{D}=0.05$ cross section. Core swirling motions have ignorable influence on the flow field of the top part in the cross sections slightly downstream of the lobed trailing edge. The flow field within the bottom part of the cross section is characterized by the swirling motions and the streamlines within the bottom part are gradually forced to the circumferential direction when the swirling angle is augmented. For the cross sections downstream of the $\mathrm{L} / \mathrm{D}=0.1$ cross section, the swirling motions within the bottom part are gradually transferring to the middle part through convective and diffusive flow. And the swirling motions are found to cause the clockwise stream-wise vortex to stretch into several smaller-scale vortexes through the drag effect. Besides, the induced smaller-scale vortexes are more influenced and strengthened by the swirling motions when case with a bigger swirling angle is investigated. 
With regard to the aerodynamic performances for the studied swirling cases, both the mixing efficiency and the total pressure loss are suggested to be nearly increased as a result of the boosted swirling angle. As for the profiles of the thermal mixing efficiency, the difference between the baseline case and the $15^{\circ}$ case can be ignored which indicates that the loss caused by the destroyed vortex pattern and the benefit induced by the improved smaller-scale vortexes are almost counteract with each other for the $15^{\circ}$ case. In the last studied cross section when compared with the baseline case, the case with a maximum swirling angle of $30^{\circ}$ has increased $6.94 \%$ for the thermal mixing efficiency and decreased $0.42 \%$ for the total pressure recovery coefficient.

\section{Acknowledgments}

The authors would like to acknowledge the anonymous reviewers who have helped to improve this paper. In addition, the authors would like to thank M.S. Mingyue Sun for helping refining the figures incorporated in this paper.

\section{References}

[1] Rao, Srisha MV, and G. Jagadeesh. "Novel supersonic nozzles for mixing enhancement in supersonic ejectors." Applied Thermal Engineering 71.1 (2014): 62-71.

[2] Liu, You-Hong. "Experimental and numerical investigation of circularly lobed nozzle with/without central plug." International Journal of Heat and Mass Transfer 45.12 (2002): 2577-2585.

[3] Shan, Yong, and Jing-zhou Zhang. "Numerical investigation of flow mixture enhancement and infrared radiation shield by lobed forced mixer." Applied Thermal Engineering 29.17 (2009): 3687-3695.

[4] MQ Zaman, K. B., F. Y. Wang, and N. J. Georgiadis. "Noise, turbulence, and thrust of subsonic freejets from lobed nozzles." AIAA journal 41.3 (2003): 398-407.

[5] PRESZ, WALTER M., BRUCE KL MORIN, and Robert G. Gousy. "Forced mixer lobes in ejector designs." Journal of Propulsion and Power 4.4 (1988): 350-355.

[6] Skebe, Stanley A., Robert William Paterson, and T. J. Barber. Experimental investigation of three-dimensional forced mixer lobe flow fields. American Institute of Aeronautics and Astronautics, 1988.

[7] Waitz, I. A., et al. "Enhanced mixing with streamwise vortex." Progress in Aerospace Sciences 33.5 (1997): 323-351.

[8] Mao, R. H., S. C. M. Yu, and L. P. Chua. "Kelvin-Helmholtz and streamwise vortexes in the near wake of a single-lobe forced mixer." Proceedings of the Institution of Mechanical Engineers, Part G: Journal of Aerospace Engineering 220.4 (2006): 279-298.

[9] Wu, Yeng-Yung Tsui, Po-Wenn. "Effects of lobe geometry on the mixing flow in multilobe mixers." Numerical Heat Transfer: Part A: Applications 39.1 (2001): 61-77.

[10] M. Yu, Simon C., T. H. Yip, and C. Y. Liu. "Mixing characteristics of forced mixers with scalloped lobes." Journal of propulsion and power 13.2 (1997): 305-311.

[11] Liu, Y. H., et al. "Effect of Lobe Numbers on the Performance of a Lobed Mixer in the Forced Mixing Exhaust System." Journal of Aerospace Power 25.8 (2010): 1683-1689.

[12] ZHAO, Jian-chao, Yi XIE, and You-hong LIU. "Effect of the Width Ratio of the Core Lobe to the Secondary Lobe on the Performance of a Lobed Mixer in a Forced Mixing Exhaust 
System." Science Technology and Engineering 34 (2010): 028.

[13] Xie, Yi, and You - hong Liu. "Numerical investigation of mixing length on performance of lobed forced mixer nozzles." Heat Transfer-Asian Research 40.3 (2011): 205-216.

[14] Mager, A. "Incompressible, viscous, swirling flow through a nozzle." AIAA Journal 9.4 (1971): 649-655.

[15] Hoffman, Joe D., H. Doyle Thompson, and DAVID L. MARCUM. "Analytical study of swirler effects in annular propulsive nozzles." Journal of Propulsion and Power 3.5 (1987): 465-466.

[16] Dutton, J. C. "Swirling supersonic nozzle flow." Journal of Propulsion and Power 3.4 (1987): 342-349.

[17] Knowles, K., and P. W. Carpenter. "Subcritical swirling flows in convergent, annular nozzles." AIAA journal 27.2 (1989): 184-191.

[18] Lei, Zhijun, et al. "Influence of Inlet Swirl on the Aerodynamics of a Model Turbofan Lobed Mixer." ASME 2010 International Mechanical Engineering Congress and Exposition. American Society of Mechanical Engineers, 2010.

[19] Lei, Zhijun, et al. "Effects of core flow swirl on the flow characteristics of a scalloped forced mixer." Journal of Engineering for Gas Turbines and Power 134.11 (2012): 111201.

[20] Lei Zhijun. "Experimental Study on the Mixing Mechanism of Lobed Mixer with Inlet Swirl in Model Turbofan Engines." Chinese Academy of Science, 2011.

[21] Xie, Yi, and You-Hong Liu. "A modified thermal mixing efficiency and its application to lobed mixer nozzle for aero-engines." Heat Transfer Research 42.4 (2011): 317-335.

[22] Du, Liwei, Youhong Liu, and Teng Li. "Numerical Predictions of Scarfing on Performance of S-Shaped Nozzle with Asymmetric Lobe." Journal of Propulsion and Power 31:2 (2014): 604618.

[23] Xie, Yi, and You - hong Liu. "Numerical investigation of lobe spacing ratio on performance of forced mixer nozzle." Heat Transfer-Asian Research 40.7 (2011): 593-607. 


\section{Table and Figure Captions}

Fig. 1 Computational domain of the investigated geometrical model …...........................................5

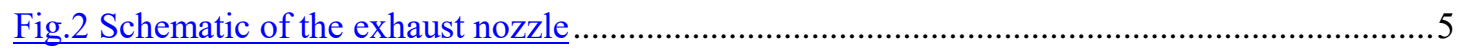

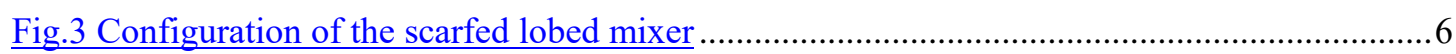

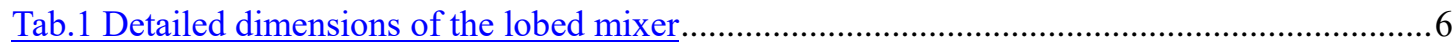

Tab.2 Element numbers of the multi-block structured grids incorporated in grid test .......................7

Fig.4 Profiles of thermal mixing efficiency for grid test.................................................................

Fig.5 Profiles of total pressure recovery coefficient for grid test................................................... 8

Fig.6 Mesh of investigated geometrical model after grid test........................................................

Tab.3 Boundary conditions used in the numerical investigations ................................................. 10

Fig.7 Comparison of normalized counter-clockwise circulations between experiment and CFD ...11

Fig. 8 Comparison of normalized clockwise circulations between experiment and CFD ...............12

Fig.9 Contour of axial velocity in the meridional plane of the lobe crest for the baseline case....... 14

Fig. 10 Contour of axial velocity in the meridional plane of the lobe valley the baseline case ........14

Fig.11 Contours of stream-wise vortexes in the cross sections for the baseline case ......................15

Fig. 12 Contour of axial velocity in the meridional plane of lobe crest for the $10^{\circ}$ case ..................17

Fig. 13 Contour of axial velocity in the meridional plane of lobe crest for the $30^{\circ}$ case...................18

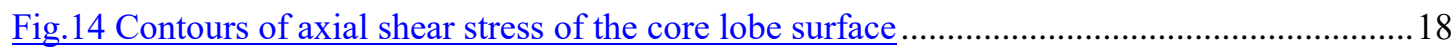

Fig. 15 Streamlines within the $\mathrm{L} / \mathrm{D}=-0.05$ cross section of the core flow field ..............................18

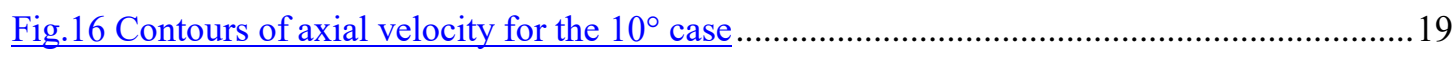

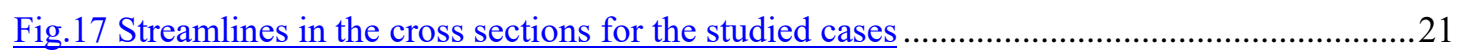

Fig. 18 Contours of stream-wise vortexes in the cross sections for the studied cases .....................21

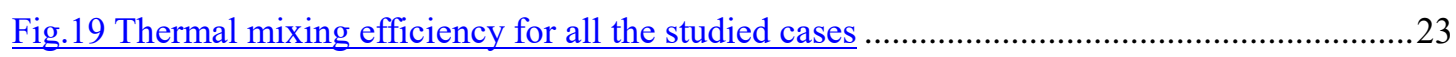

Fig.20 Total pressure recovery coefficient for all the studied cases............................................23 